\title{
Thermogravitational and hybrid convection in an obstructed compact cavity
}

\author{
Marcello Lappa and Saad Inam ${ }^{1}$ \\ ${ }^{1}$ Department of Mechanical and Aerospace Engineering, University of Strathclyde, James Weir \\ Building, 75 Montrose Street, Glasgow, G1 1XJ, UK - email: marcello.lappa@strath.ac.uk
}

\begin{abstract}
The flow over a topography or a step is a fundamental problem in fluid dynamics with relevance to many fields and circumstances. In the present analysis direct numerical simulation (DNS) is used to examine the properties of the thermofluid-dynamic field in a square cavity with a heated obstruction located on the bottom. The involved dynamics include forced flow driven by injection of cold fluid through an orifice and the buoyancy convection of thermal origin, which naturally emerges in the considered cavity as a result of the prevailing temperature gradients. The relative importance of these mechanisms is assessed by considering different values of the Rayleigh number (spanning several orders of magnitude) and using a peculiar analysis hierarchy where selected effects are intentionally switched on or off depending on the targeted regime. In particular, first, attention is paid to pure thermogravitational convection driven by the presence of vertical and horizontal heated surfaces (the hot sides of the obstruction); then, the hybrid regime is examined where buoyancy convection is modulated by currents of forced flow for values of the Richardson number $\cong 1$. We also explore the resulting patterning behavior for different thermal and kinematic boundary conditions (distinct thermal behaviors of the top wall and alternate positions of the inflow and outflow sections). For each case, the Nusselt number relating to the vertical and horizontal sides of the obstruction is evaluated and put in relation with the flow topology. The study is also supported by analysis of the frequency spectrum for the situations in which relatively chaotic states emerge.
\end{abstract}

Key words: Buoyancy flow, water, heat exchange, instability and bifurcation in fluid dynamics.

\section{Introduction}

Fluid vorticity is ubiquitous in typical problems involving thermal convection, where it is typically produced in the form of steady or unsteady rolls and associated thermal plumes [1].

However, localized vorticity is also common in many isothermal technical and industrial applications where it can be induced by blunt obstacles. This is the case for instance of pipe systems in chemical plants or food-processing industries. Related hydraulic circuits are often featured by sudden changes in the cross-sectional area and/or other kinds of obstructions mounted in a direction perpendicular to the prevailing current (e.g., ribs and baffles, which prevent the flow from developing a regular path). Other relevant examples can be found in the very general area of fluid machinery where flow separation typically leads to an increase in the frictional shear stress (thereby causing significant energy losses, Hattori and Nagano [2]). This subject also displays notable kinships with other important engineering challenges such as the determination of the pressure and 
shear stress distribution over ground vehicles (Dai et al. [3]; Redchyts et al., [4]) or the production of wind energy (Sherry et al. [5]).

For all these reasons, the general problem relating to the topology and nature (steady, oscillatory or turbulent) of flows over and around wall-mounted obstacles (or in the presence of geometrical contractions or orifices) has long attracted the interest of researchers.

In particular, an archetypal configuration for studying these fluid-dynamics behaviors that has received particular attention over the last two decades is the flow over a forward-facing step (FFS). This geometrical model has been conceived by investigators as a simplified academic configuration especially suited to assess the response of the flow ahead of blunt obstacles to well-controlled changes in the geometric parameters. Investigations along these lines are, for instance, those by Refs [5-12].

By virtue of these studies (and references therein) it has been clarified that forced flow in ducts affected by shrinkage in the cross-sectional area can be very complex, often hiding flow physics yet to be completely understood. Moreover, there is some general consensus that the dynamics are more involved than the equivalent ones relating to the companion (mirror image) geometry with a backward-facing step [13-15].

Apart from the interest that attaches to these dynamics from a purely (fundamental) fluid-dynamic point of view (especially for the non-linearities embedded in the model equations which make the transition from laminar to turbulent conditions a not-yet-solved problem [16]), these subjects have attracted significant interest also due to their relevance to engineering applications involving heat transfer. Indeed, the significant mixing between colder and warmer fluid that occurs in the abovementioned recirculation regions can have a considerable impact on the thermal performance of many devices. Forward and backward facing step flows can be found in chemical processes and energy systems equipment, environmental control systems, heat exchangers of various types, cooling systems for electronic devices and nuclear reactors, combustion chambers and cooling passages in turbine blades (see, e.g., Sun and Jaluria [17]; Arrif et al. [18]; Nadjib et al., [19]; Lappa[20]). The magnitude of heat exchange in all these systems also depends to a significant degree on the spatio-temporal behavior of the flow, which explains why some studies have concentrated on the laminar regime (Abu-Mulaweh et al. [21]; Abu-Mulaweh [22]), while others have specifically addressed the turbulent one (Moosavi and Nassab [23]; Nassab et al. [24]; Oztop et al. [25]) and/or on the related transition process (Xie and Xi [26]).

Though all these valuable analyses have significantly contributed to put together the pieces of the puzzle relating to understanding heat transfer in these systems, most surprisingly, only a handful of them have expressly taken into account thermal buoyancy. This effect still raises many fundamental (unsolved) questions.

To the best of our knowledge, some useful information along these lines can be found in the experimental study by Abu-Mulaweh et al. [21]. These authors specifically concentrated on the horizontal FFS, the reader being also referred to the masterful exposition given later by AbuMulaweh [22]. Over the considered range of parameters (Reynolds number and Grashof numbers 
based on the step height varying in the intervals, $\mathrm{O}\left(10^{2}\right) \leq \operatorname{Re}_{h} \leq \mathrm{O}\left(10^{3}\right)$ and $\mathrm{O}\left(10^{3}\right) \leq G r_{h} \leq \mathrm{O}\left(10^{4}\right)$, respectively), it was found that the size of the recirculation regions increases while the heat transfer rate from the heated downstream wall decreases as the step becomes ticker (moreover, both quantities grow as the inlet velocity increases). They also observed that in some circumstances the upper recirculation area can be suppressed, while in other cases disturbances promoting transition from laminar conditions to turbulent flow start to grow just in this region.

Other interesting (partially relevant) information can be found in existing numerical studies about the typical instabilities of buoyancy and hybrid thermocapillary-thermogravitational convection in crucibles with different shapes used for the production of semiconductor or opto-electronic materials (see, e.g., [27-29]) or other analyses dealing with building heating and ventilation or electronic devices cooling (Sun and Jaluria [17]; Yarin [30]; Venkatasubbaiah and Jaluria [31]; Morsli et al. [32]; Kachi et al. [33]).

As the reader might have realized at this stage, problems involving fluids flowing over steps can indeed support a multifaceted spectrum of different research initiatives and are omnipresent in technical and engineering applications. As implicitly made evident by the focused review of literature reported in this introduction, however, a significant lack of information seems to still exist with regard to conditions for which forced flow and thermal (buoyancy) convection compete in determining the patterning behavior and the evolutionary progress of these systems towards chaos. In order to fill some existing gaps, in particular, in the present study we consider both situations in which thermogravitational flow is dominant $(\mathrm{Re}=0)$ or it competes with a forced flow having a similar strength (these circumstances formally correspond to values of the so-called Richardson number, defined as ratio of the Grashof number and the square of the Reynolds number, approaching the unit order of magnitude.). Moreover, since most of existing studies have assumed horizontally elongated cavities or ducts, here insights are sought from consideration of another archetypal setting, i.e. a compact geometry, able to support at the same time forced flow and buoyancy convection of the Rayleigh-Bénard or Hadley type. Explored degrees of freedom concern the magnitude of the Rayleigh number and the boundary conditions assumed for temperature and velocity (the reader being referred to Sect. 2 for additional details about these forms of convection, related characteristic numbers and the explored intervals).

\section{Mathematical and Numerical model}

\subsection{The Geometry and related boundary conditions}

The considered configuration is shown in Fig. 1. It consists of a square two-dimensional cavity with a square obstruction located on the bottom. The cavity is also equipped with inflow and outflow sections. More precisely as evident in the sketch, while the outflow section is located at mid-height between the horizontal hot surface of the obstacle and the top wall of the cavity, two different 
distances from the floor are possible for the inflow section (the fluid being injected through one 'or' the other in order to force it to follow different paths).

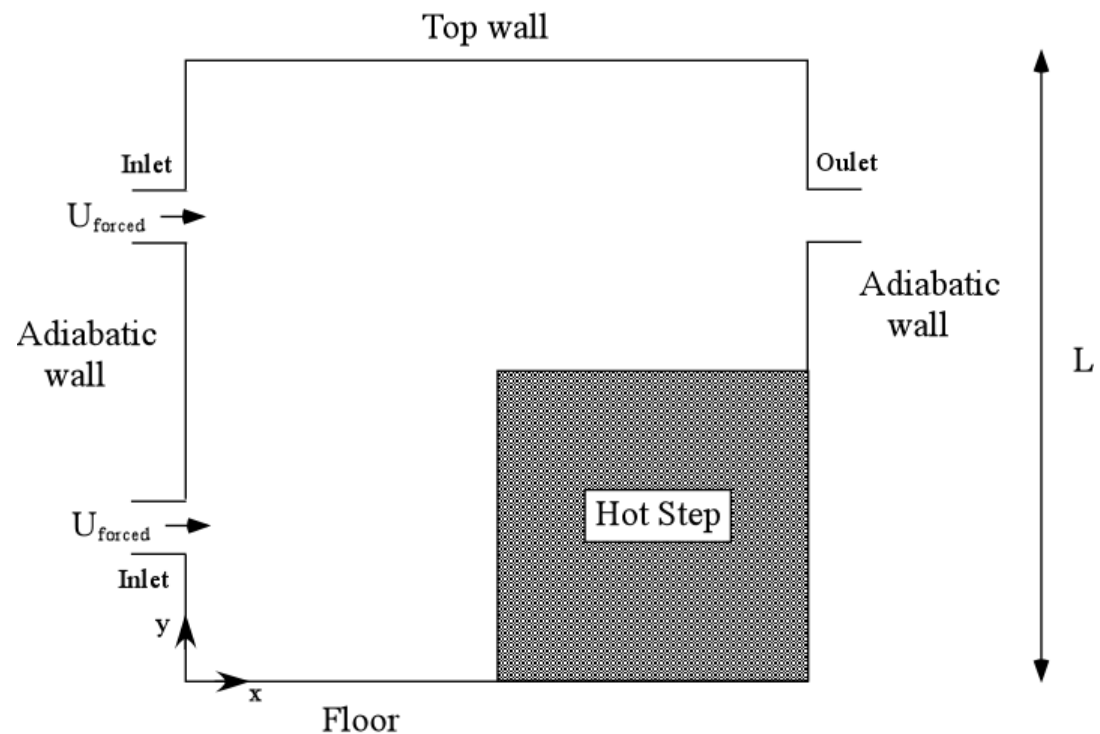

Figure 1: Sketch of the considered geometry and related kinematic and thermal boundary conditions.

By setting the fluid injection velocity $U_{\text {forced }}$ to zero, we implicitly assume that no fluid enters or leaves the cavity (formally corresponding to the situation for which the inflow and outflow sections are replaced by portions of solid wall).

From a thermal point of view, while the sidewalls are considered adiabatic for all cases examined, the floor is assumed either adiabatic or at constant temperature (namely, set at the same temperature $T_{\text {hot }}$ of the obstacle). A different concept is implemented for the top wall, which is considered cold ( $T_{\text {cold }}$ ) and isothermal for $U_{\text {forced }}=0$ (pure buoyancy convection) or adiabatic when $U_{\text {forced }} \neq 0$ (hybrid convection). In the latter case the cooling role of the top wall is transferred to the fluid being injected into the cavity (which has velocity and temperature set to $U_{\text {forced }}$ and $T_{\text {cold, }}$, respectively).

\subsection{Governing equations and characteristic numbers}

Practical governing equations for the considered problem in non-dimensional form can be obtained by referring velocity and pressure to proper scales. Using $\alpha / L$ as velocity scale (where $\alpha$ is the fluid thermal diffusivity), the reference quantities for pressure and time follow naturally as $\rho \alpha^{2} / L^{2}$ and $L^{2} / \alpha$, respectively (where $\rho$ is the fluid density). The non-dimensional continuity and momentum equations read accordingly:

$\underline{\nabla} \cdot \underline{V}=0$

$\frac{\partial \underline{V}}{\partial t}=-\underline{\nabla} p-\underline{\nabla} \cdot[\underline{V} \underline{V}]+\nabla^{2} \underline{V}-\operatorname{Pr} R a T \underline{i}_{g}$ 
where

$$
R a=\operatorname{Pr} G r=\frac{g \beta_{T} \Delta T L^{3}}{v \alpha}
$$

is the classical Rayleigh number based on the imposed temperature difference and the fluid thermal expansion coefficient $\beta_{T}$ (used to link the variations of density to the corresponding variations of temperature in the frame of the so-called Boussinesq approximation); moreover, $v$ is the kinematic viscosity of the considered fluid (the ratio $v / \alpha$ being the well-known Prandtl number Pr, set to 1 in the present work). Introducing the nondimensional temperature as the ratio between the local temperature minus a reference temperature $\left(T_{\text {cold }}\right)$ and the imposed $\Delta T$, the nondimensional energy equation can be cast accordingly in compact form as

$$
\frac{\partial T}{\partial t}+\underline{\nabla} \cdot[\underline{V T}]=\nabla^{2} T
$$

Problem closure, however, also requires a mathematical formulation of the behavior of velocity and temperature at the system boundaries. Such a need leads to the introduction of another characteristic number, i.e. the Reynolds number relating to the velocity with which the fluid is injected into the cavity. This number simply reads:

$$
\operatorname{Re}=\frac{U_{\text {forced }} L}{v}
$$

As outlined in the introduction, for practical reasons, the Rayleigh and Reynolds numbers can be combined into another typical nondimensional parameter, i.e. the so-called Richardson number:

$$
R i=\frac{g \beta_{T} \Delta T L}{U_{\text {forced }}^{2}}=\frac{R a}{\operatorname{Pr}^{2} \mathrm{e}^{2}}
$$

This additional parameter has enjoyed a widespread use in past works on these subjects given its intrinsic ability to measure the relative importance of buoyancy and forced convection. The situations with pure buoyancy convection or forced flow are obviously recovered in the limit as the Richardson number tends to infinite or zero, respectively. As outlined in the introduction, the specific case with $\mathrm{Re}=0$ (i.e. the closed cavity) is also of special interest because, even though forced flow is absent, fluid motion is brought about by different coexisting mechanisms. Buoyancy convection of thermal nature can indeed behave in very different ways according to whether it originates from heated surfaces which are horizontal or vertical. In the former case, the emerging 
flow falls under the general heading of Rayleigh-Bénard (RB) convection and its variants (which so much attention has attracted over the last decades owing to its important implications in the study of chaotic systems, see, e.g., Gollub and Benson [34]). In the latter case, the much more stable Hadley convection is produced (e.g., Gershuni [35]).

Even though the driving force is the same, the hierarchy of bifurcations displayed by purely buoyant flow can change significantly depending on whether the prevailing temperature difference is parallel or perpendicular to gravity (i.e. according to whether the target problem is of the RB or Hadley type, see, e.g., Chapters 4 and 6 in [1]). The situation becomes more complex when both convective mechanisms are present at the same time and, obviously, an additional degree of complexity is brought in if the considered heated surfaces are also subjected to an imposed forced flow (a third independent convective mechanism).

\subsection{Boundary conditions}

The non-dimensional thermal boundary conditions (BCs) for the considered problems can be summarized as follows:

Side walls $(\mathrm{x}=0$ or $\mathrm{x}=1): \partial T / \partial \mathrm{x}=0$

Step walls $(\mathrm{x}=1 / 2,0 \leq \mathrm{y} \leq 1 / 2$ and $\mathrm{y}=1 / 2,1 / 2 \leq \mathrm{x} \leq 1) T=1$

Floor $(\mathrm{y}=0,0 \leq \mathrm{x} \leq 1 / 2), \partial T / \partial \mathrm{y}=0$ (adiabatic)

or $T=1$ (isothermal)

Top wall $(\mathrm{y}=1,0 \leq \mathrm{x} \leq 1) T=0$ (isothermal, $\mathrm{Re}=0$ ) or $\partial T / \partial \mathrm{y}=0$ (adiabatic, $\mathrm{Re} \neq 0$ )

We define accordingly the Nusselt number relating to the vertical and horizontal walls of the step as:

$N u_{\text {step }}^{\text {vert }}=-2 \int_{0}^{1 / 2} \frac{\partial T}{\partial x} d y$ and $N u_{\text {step }}^{\text {horiz }}=2 \int_{1 / 2}^{1} \frac{\partial T}{\partial y} d x$

From a kinematic point of view, all the walls are considered as no-slip surfaces, which means that the two velocity components along $\mathrm{x}$ and along $\mathrm{y}(u, v)$ are formally set to zero there. For the cases with $\mathrm{Re} \neq 0$, additional conditions are required at the inflow and outflow section (assumed to have a non-dimensional vertical extension $l=0.1)$. For the inflow section we have simply set:

Inflow $(\mathrm{x}=0,0.2 \leq \mathrm{y} \leq 0.3$ or $0.7 \leq \mathrm{y} \leq 0.8): u=U_{\text {forced }}, v=0, T=0$

A more involved rationale has been used to define the relevant BCs for the outflow section. Determining proper conditions for open boundaries generally requires some special care to ensure 
that no spurious oscillations are generated, which can jeopardize the physical consistency of the entire velocity (and temperature) field and cause numerical instabilities. Existing studies on the subject have led to a common approach nowadays known as the "open boundary condition" (OBC) framework (Hattori et al. [36]; Dong et al. [37]). With such approach, in place of simply spatial conditions (such as those used for the inflow section) more involved spatio-temporal conditions are used, generally cast in mathematical form via the so-called "prognostic equation":

Outflow $(\mathrm{x}=1,0.7 \leq \mathrm{y} \leq 0.8): \frac{\partial u}{\partial t}+D \frac{\partial u}{\partial x}=0, \frac{\partial v}{\partial t}+D \frac{\partial v}{\partial x}=0, \frac{\partial T}{\partial t}+D \frac{\partial T}{\partial x}=0$

namely, a transport equation where $D$ is a constant assumed to be equal to the averaged velocity perpendicular to the boundary $D=\underline{V} \cdot \underline{\hat{n}}$. This modus operandi has been proven to provide reasonable algorithm stability and guarantee the physical consistency of the solution by preventing the propagation of non-physical fluctuations of pressure and/or temperature in the upstream direction.

\subsection{The Numerical method}

The equations reported in Sect. 2.2 and related boundary conditions listed in Sect. 2.3 have been solved using the OpenFoam computational platform in the framework of the PISO (Pressure Implicit Split Operator) technique proposed by Issa [38] in its original form.

The considered method may be regarded as a spin-off of the so-called Hodge decomposition theorem (see, e.g., [39]); it states that any vector field can be expressed as the combination of a divergence-free contribution and the gradient of a scalar potential (a curl-free part). As a consequence, a velocity field can be considered fixed when its curl (vorticity) and divergence (and normal component at the boundary) are known.

This splitting becomes particularly useful if one considers that the typical evolution of an incompressible flow is generally mediated or governed by the specific interplay or coupling that is established between pressure and velocity (the so-called "primitive variables"). While only the velocity appears in the continuity equation, i.e. eq. (1) (from a mathematical point of view this equation expresses that this vector field must be solenoidal for an incompressible flow), both $\underline{V}$ and $p$ are present in eq. (2). Notably, this property is at the basis of many techniques [40-44], generally known as projection methods, where the momentum equation is used to define a time-marching procedure for the solution of the entire problem relating to the thermo-fluiddynamic field. These methods take advantage of the specific differential form of the pressure in this equation (it appears inside a gradient, i.e. $\underline{\nabla}$ ). Recalling that the gradient of a scalar quantity is always curl-free this leads to the remarkable realization that the vorticity associated with the velocity field does not depend directly on the pressure. It is therefore possible to determine a field that has the same curl as the 'physical' velocity field by dropping the pressure gradient and solving the simplified version of 
the momentum equation obtained accordingly. This approach formally yields a velocity field, which given its unphysical nature, is generally referred to as the "intermediate velocity" field $\underline{V}^{*}$.

Making it physically consistent and fixed from a mathematical point of view (in the sense that has been given to this adjective above) requires making sure that the field also possesses the right divergence. For the incompressible Navier-Stokes equations, this is equivalent to enforcing the soleinodal nature of the velocity field. i.e. $\underline{\nabla} \cdot \underline{V}=0$. With the PISO method this is achieved, by expressing the physical velocity as $\underline{V}=\underline{V}^{*}-\xi \underline{\nabla} \mathrm{p}$ (where $\xi$ is a constant) and substituting it into the continuity equation. This formally leads to an elliptic equation where the unknown is the pressure field. Once determined in this way, the pressure can be used to correct the intermediate velocity field. The entire process turns around the intermediate velocity field $\underline{V}^{*}$, which formally satisfies the aforementioned Hodge theorem (as formally $\underline{V}^{*}$ can be seen as $\underline{V}^{*}=\underline{V}+\xi \underline{\nabla} \mathrm{p}$, i.e. the linear combination of a divergence-free contribution and the gradient of a scalar potential).

With OpenFoam such algorithm is implemented in the framework of a collocated grid approach, i.e. all the variables are defined in the center of the cells and a special interpolation of the velocity is employed on the cell faces in order to guarantee good coupling of velocity and pressure [45].

Moreover, for the present study, we have used second order central difference schemes to discretize the velocity and temperature equations with regard to both convective and diffusive terms. The resulting system of algebraic equations has been treated using a Preconditioned Bi-Conjugate Gradient (PBiCG) with an Incomplete Lower Upper (DILU) preconditioner in the predictor step, while the solution of the elliptic pressure equation has been addressed in the frame of a Generalized Geometric-Algebraic Multi-Grid (GAMG) method. Although 2D simulations may not capture the underlying physics quantitatively, our findings may support the clarification of controversial experimental observations and eventually lead to the identification of new physical mechanisms.

\subsection{Algorithm Validation}

The overall algorithm has been validated through comparison with available results in the literature. In particular, its performances have been tested with respect to both types of thermal convection being present in the problem considered here, namely, RB and Hadley flow. The outcomes of the dedicated simulations we have conducted in this regard are summarized in Tables I and II, respectively.

Table I: $\mathrm{A}=1$, Rayleigh-Bénard convection, $R a=10^{5}, \mathrm{Pr}=0.71$. Comparison with Table 2 of Ouertatani et al. [46], mesh $128 \times 128$. Velocity scaled with $\mathrm{u}_{0}=\operatorname{sqrt}\left(\mathrm{g} \beta_{T} \mathrm{H} \Delta T\right)$.

\begin{tabular}{ccc}
\hline \hline Quantity & Present & Ouertatani et al. [46] \\
\hline \hline $\mathrm{U}_{\max }$ & 0.3443 & 0.3442 \\
$\mathrm{~V}_{\max }$ & 0.3754 & 0.3756 \\
$N u_{h}$ & 3.9204 & 3.9097 \\
\hline \hline
\end{tabular}




\subsection{Mesh resolution and Kolmogorov length scale}

The choice of an adequate mesh when the problem is approached in the framework of DNS (Direct Numerical Simulation) is not as straightforward as one would imagine. In general, two requirements must be satisfied in order to make the simulations reliable.

First of all, a parametric investigation must be carried out to make sure that the solution does not depend on the considered grid. This implies testing the percentage variation experienced by some representative fluid-dynamic quantities when the density of the mesh is increased. If simulations are carried out for values of the characteristic numbers (e.g., $R a$ ) spanning several orders of magnitude, this refinement study must be conducted for each order of magnitude considered or, as an alternative, the mesh which makes the solution grid-independent obtained for the highest considered value of Ra must be used for all the cases (this modus operandi being the most expensive in terms of computational cost or efficiency).

Table II: Hadley flow, $R a=10^{6}, \operatorname{Pr}=0.71, \mathrm{~A}=1$, mesh 100x100 (Legend: $|\psi|_{\max }$ - Maximum absolute value of the stream function, $\mathrm{v}_{\max }$ - Max vertical velocity component on a horizontal mid-plane, $\mathrm{x}_{\max }$ - Position of $v_{\max }, u_{\max }$ - Max horizontal component on a vertical mid-plane, $y_{\max }$ - Position of $u_{\max }$. $N u_{h o t}$ - Average Nusselt number on the hot boundary. $N u_{\max , h o t}$ - Maximum Nusselt number on the hot boundary, $\mathrm{y}_{\mathrm{Nu}, \max }$ - Position of $N u_{\max }, h o t, N u_{\min , h o t}$ - Minimum Nusselt number on the hot boundary, $\mathrm{y}_{\mathrm{Nu}, \min }$ - Position of $\left.N u_{\min , h o t}\right)$.

\begin{tabular}{ccc}
\hline \hline Parameter & present & De Vahl Davis and Jones [47] \\
\hline \hline$\left.\psi\right|_{\max }$ & 16.919 & 16.750 \\
$\mathrm{~V}_{\max }$ & 216.09 & 219.36 \\
$\mathrm{x}_{\max }$ & 0.0368 & 0.0379 \\
$\mathrm{u}_{\max }$ & 64.97 & 64.63 \\
$\mathrm{y}_{\max }$ & 0.848 & 0.850 \\
$N u_{\text {hot }}$ & 8.913 & 8.817 \\
$N u_{\max , h o t}$ & 18.193 & 17.925 \\
$\mathrm{y}_{\mathrm{Nu}, \max }$ & 0.0368 & 0.0378 \\
$N u_{\min , h o t}$ & 0.970 & 0.989 \\
$\mathrm{y}_{\mathrm{Nu}, \min }$ & 1 & 1 \\
\hline \hline
\end{tabular}

As a second requirement, if the considered flow is in turbulent conditions, the size of the mesh must be comparable (or equal) to the so-called Kolmogorov length scale $(\zeta)$, i.e. the smallest flow scale present in the considered problem.

Estimates about this quantity can be found in the literature for different circumstances.

For forced flow, it can conveniently be evaluated (Pope [48]) as: 
$\zeta_{\mathrm{Re}} \cong \mathrm{Re}^{-3 / 4}$

This correlation should obviously be used when $R i<\mathrm{O}(1)$ (Coceal et al. [49]), whereas, in the opposite limiting condition in which thermal convection is the most important convective mechanism (namely, $R i>\mathrm{O}(1)$ ) alternate estimates should be used. These, in turn, can change depending on the relative direction of the prevailing temperature gradient and gravity. As explained in Sect. 2.2, indeed, the progression of thermal convection towards turbulence and its spatial and temporal features can be markedly different according to whether convection originates from a heated horizontal or vertical wall.

In the former case, following Kerr [50] and De et al. [51]:

$\zeta_{R a \|}=1.3 R a^{-0.32}$

while for what concerns the other situation with prevailing horizontal gradients of temperature, a quantitative measure of such a length scale can be found in the works by Paolucci [52] and Farhangnia et al. [53]:

$\zeta_{R a \perp}=\pi\left(\frac{16 \operatorname{Pr}}{R a}\right)^{3 / 8}$

When circumstances where multiple mechanisms of convection coexist are the main subject of the analysis as in the present case, the smallest possible value of $\zeta$ should be considered (see, e.g., Lappa and Gradinscak [54]). For the conditions examined here $\left(10^{3} \leq R a \leq 10^{8}, 33 \leq R e \leq 10^{4}\right)$, it is easy to verify that the smallest possible scale always corresponds to eq. (15). This equation has therefore been used to determine the size of the mesh in the most demanding conditions (i.e. $R a \geq 10^{7}$ ). For smaller values of $R a$, the grid density has been decided on the basis of a case-by-case preliminary mesh refinement assessment, as further discussed in the following sections.

\section{Results}

As anticipated in the introduction, we have explored the response of the system sketched in Fig. 1 with respect to different kinematic and thermal degrees of freedom (namely, the absence or presence of cold fluid being injected into the cavity at different heights and different possible thermal behaviors of the top and bottom walls).

However, it is worth recalling that another important degree of complexity is brought in by the development of thermal plumes. Indeed, over the range of values of the Rayleigh number considered in the present work, plumes pertaining to different regimes can be formed and influence with their properties the overall flow dynamics. Precise information along these lines can be found 
in the study by Hier Majumder et al. [55], who categorized thermal plumes on the basis of the related amount of vorticity and the gradient of temperature associated with their stem and cap. These features greatly depend on the considered fluid (Prandtl number) and the Rayleigh number. In particular, for the value of the Prandtl number assumed in the present work $(\operatorname{Pr}=1)$, plumes evolve from the so-called DV (Diffusive-Viscous) regime into the IVND (Inviscid-Nondiffusive) regime as soon as the Rayleigh number exceeds $R a \cong 10^{6}$ [1].

Given these premises, in the following, first we discuss the cases for which the cavity has no inflow and outflow sections (Sect. 3.1) where the dynamics are expected to be entirely driven by the buoyancy effects and thermal plumes; then, we move to situations where significant forced flow can cause alterations in the flow topology and related evolution towards chaos (Sects. 3.2 and 3.3).

\subsection{Closed cavity}

As already explained in Sect. 2.6, for each fundamental case a mesh refinement study was carried out to identify the minimum grid required to make the solution mesh-independent. As expected, this resulted in the need to increase the number of points with the considered value of the Rayleigh number. Related information is summarized in Tables III and IV, where we have reported for each case (in an ordered fashion): the value of $R a$, the corresponding density of the mesh for which we found the solution to be grid-independent, the nondimensional (total) heat flux through the horizontal and vertical walls of the step (the hot obstruction) and, finally, the nature of the observed regime.

Table III: Cases examined for the case of pure buoyancy convection with adiabatic bottom wall

\begin{tabular}{ccccc}
\hline \hline$R a$ & Mesh Size & $N u_{\text {step }}^{\text {horiz }}$ & $N u_{\text {step }}^{\text {vert }}$ & Regime \\
\hline \hline $10^{3}$ & $40 \times 40$ & 1.21997 & 2.42394 & Steady \\
$10^{4}$ & $40 \times 40$ & 2.24234 & 2.03534 & Steady \\
$10^{5}$ & $80 \times 80$ & 3.6756 & 6.0646 & Steady \\
$10^{6}$ & $100 \times 100$ & $\cong 4.17$ (average) & $\cong 11.7$ (average) & Single-frequency \\
$10^{7}$ & $270 \times 270$ & $\cong 5.74$ (average) & $\cong 20.3$ (average) & Single-frequency \\
$10^{8}$ & $500 \times 500$ & $\cong 11.53$ (average) & $\cong 32.2$ (average) & Weakly Turbulent \\
\hline \hline
\end{tabular}

Following the approach described in Sect. 2.3, in particular, we concentrate on two aspects, namely, the hierarchy of convective and thermal structures which are produced within the cavity as the $R a$ number increases, and, the differences in terms of patterning behavior that are developed when the thermal boundary condition at the bottom floor is changed (floor being assumed to be adiabatic or kept at constant temperature as formalized by eqs. (9a) and (9b), respectively). 
Accepted for publication in Int. J. Thermal Science, (ISSN: 1290-0729) on 11 May 2020
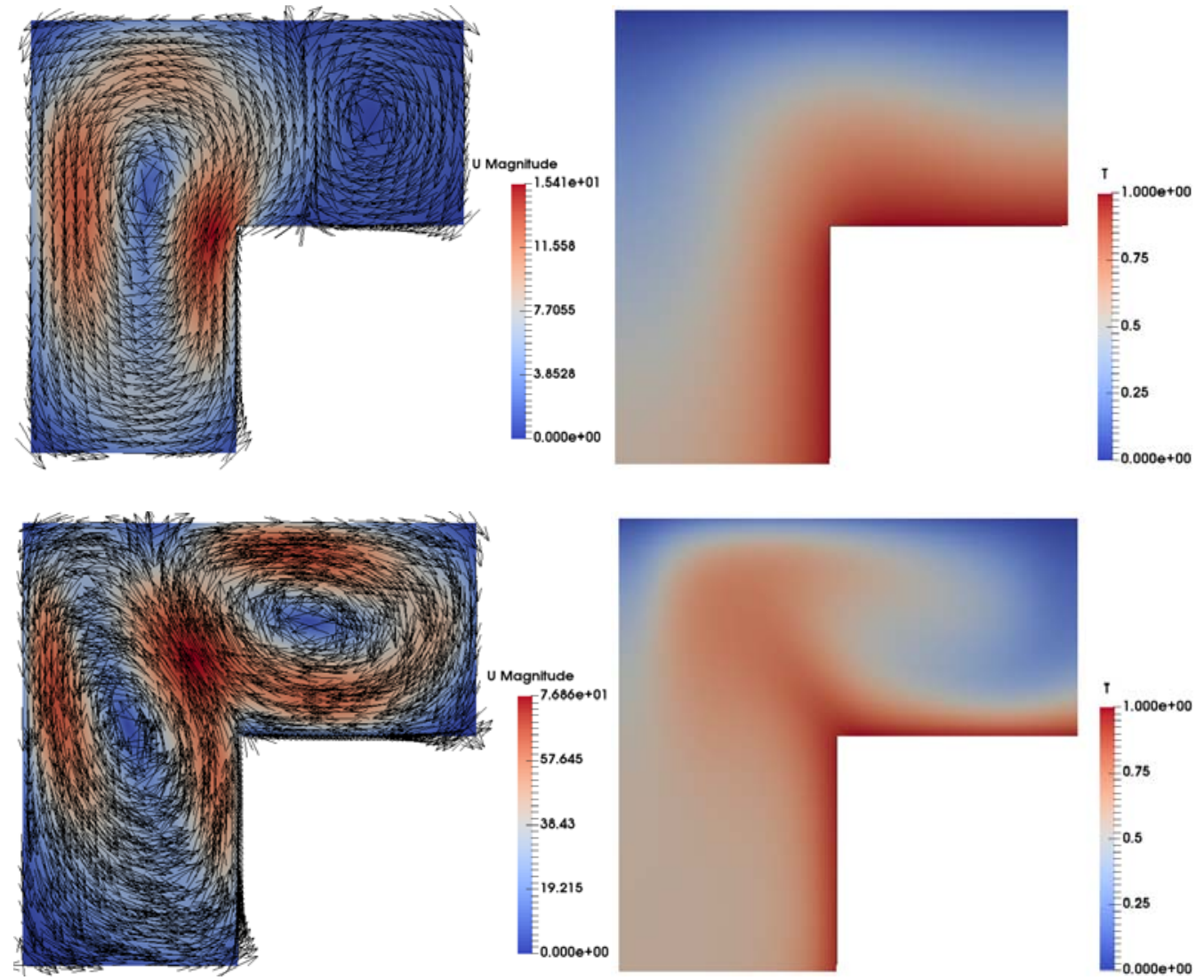

(a)
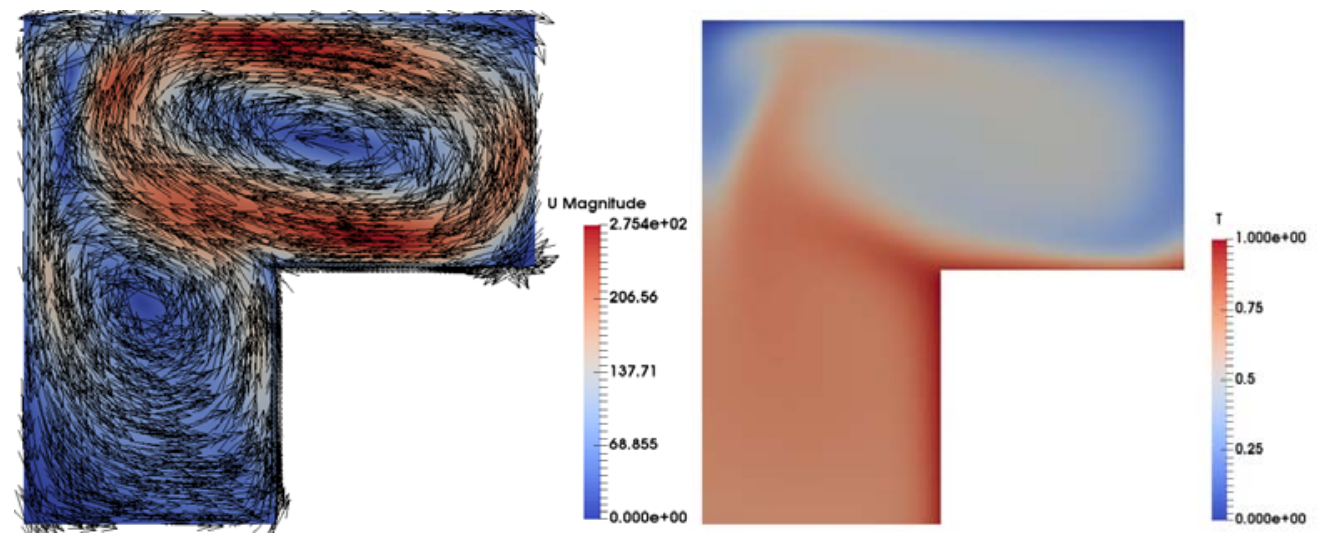

(b)
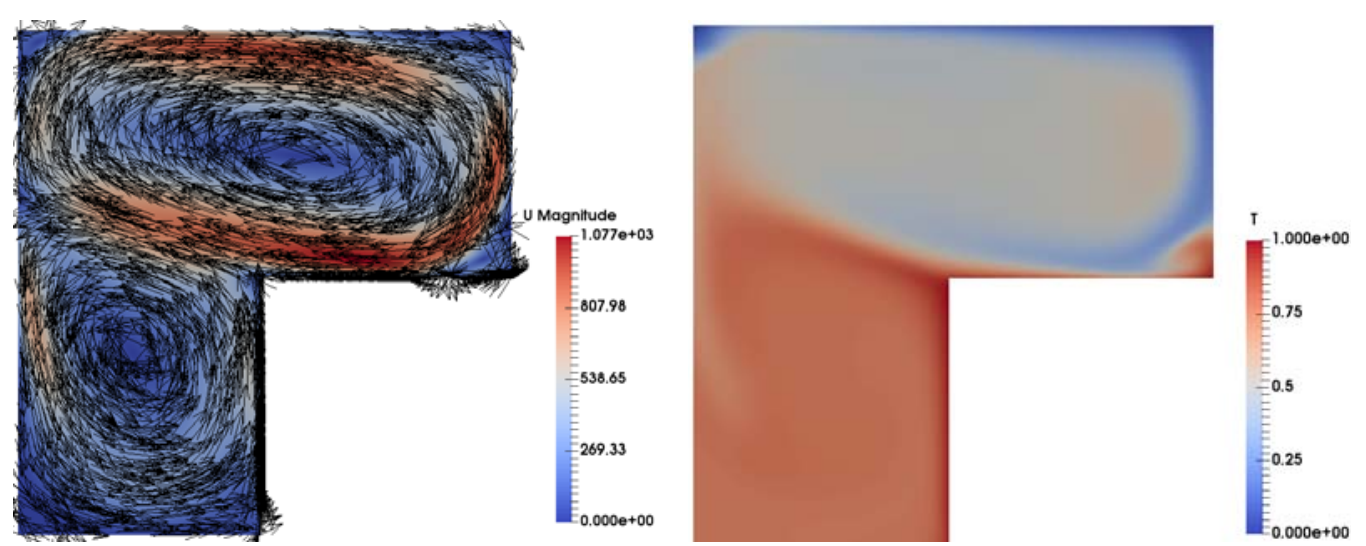

(c)

Figure 2: Snapshots of velocity field (left) and temperature distribution (right) for the case of pure buoyancy convection and cavity with adiabatic bottom wall: a) $R a=10^{4}$, b) $R a=10^{5}$, c) $R a=10^{6}$, d) $R a=10^{7}$. 


\subsubsection{Patterning Behavior for the cavity with adiabatic floor}

Most conveniently, first we discuss the cases with adiabatic floor for different values of the Rayleigh number and then examine the differences produced by an increase in the buoyancy effect due to a change in the thermal boundary condition.

As quantitatively substantiated by Figures 2-4, a growth of the (non-dimensional) velocity can be noticed for all cases as $R a$ becomes higher. Very interesting modifications, however, can also be spotted in terms of structure of the flow (i.e. morphology and extension of the rolls or vortices) established inside the cavity and related temporal behavior (i.e. evolution in time).

Along these lines, we start form the simple remark that the velocity magnitude pattern within the cavity is very similar for $R a=1 \times 10^{3}$ (not shown) and $R a=1 \times 10^{4}$ (Fig. 2(a)). Indeed, for both values of the Rayleigh number the flow is steady and one main oval-shaped vertical velocity roll is formed. This roll develops in the lower third of the cavity (between the left sidewall and the vertical side of the step) and extends vertically into the upper section of the fluid domain. The highest velocity area is located on the right hand side of the roll near the top left corner of the obstruction (the step). As evident in Fig. 2(a), the highest temperature occurs in the region surrounding the step (where, as expected, a relatively thick thermal boundary layer is established) and the temperature then gradually decreases towards the floor, the left hand side wall and the ceiling of the cavity.

Interestingly, for $R a=1 \times 10^{5}$ (Fig. 2(b)) the complexity of the velocity field increases as two ovalshaped convection rolls having a comparable magnitude can be seen at the same time. The flow is still steady and one roll is confined in the upper portion of the domain (it is located above the step), while the other originates from the lower third of the cavity. It can also be noticed that for this value of $R a$, the temperature changes occurring in proximity to the step become much more pronounced. A thin thermal (hot) boundary layer, clearly recognizable, develops along the horizontal heated wall of the obstruction and along its vertical heated wall (the two sides of the step). A thermal (cold) boundary layer is also formed close to the top (cooled) wall. Moreover, a mushroom like shape plume appears in the cavity originating from the left corner of the step and protruding towards the left top corner (plume oriented along a diagonal direction).

For $R a=1 \times 10^{6}$ (Fig. 2(c)), the boundary layers become even more pronounced (which is in agreement with known scaling laws, see, e.g., [56, 57]). Similarly, the stem of the plume is narrower than in the previous situation. Moreover, it can be seen that a secondary plume originates from the main plume cap.

The most striking difference with respect to the previous cases, however, is the nature of the flow, which becomes time-dependent. A related frequency spectrum analysis (performed using the signal of a 'numerical probe') can be seen in Fig. 3. From this we infer that this is a time-periodic case in which all the frequencies are multiples of a fundamental one. 


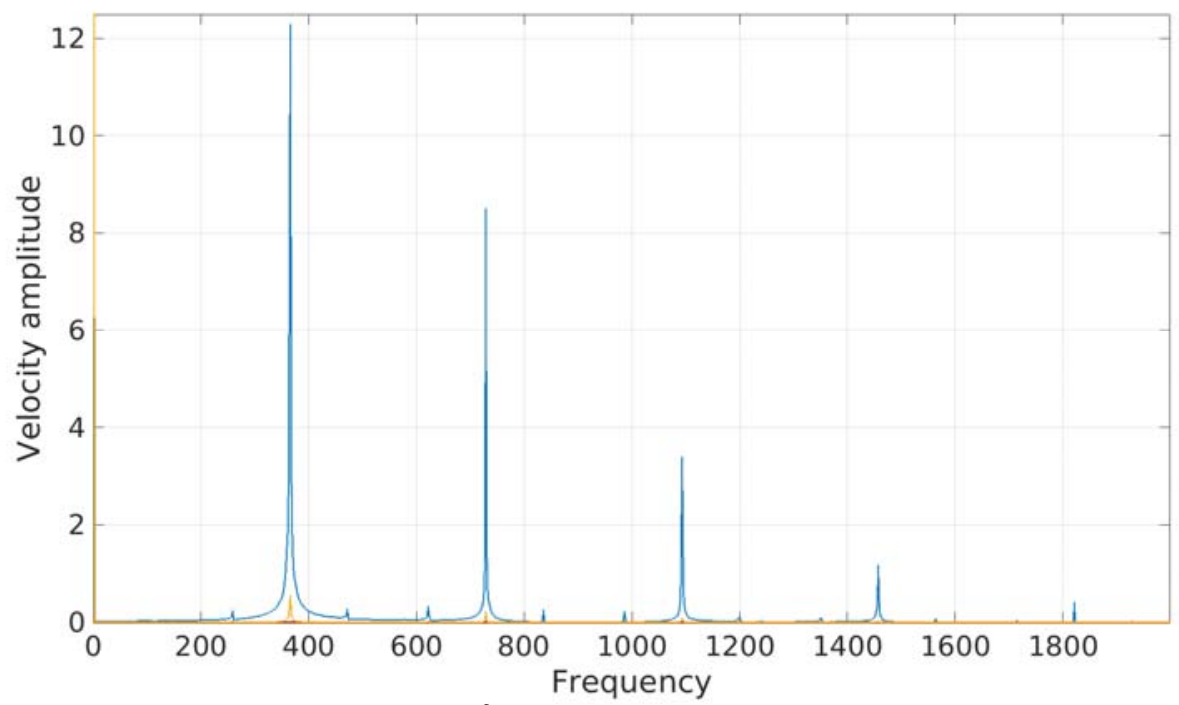

Figure 3: Frequency Spectrum $\left(R a=1 \times 10^{6}\right.$, closed cavity with adiabatic floor, signal probe located at $\mathrm{x}=0.25$ and $\mathrm{y}=0.75$ ).

If the Rayleigh number is increased to $R a=1 \times 10^{7}$ (Fig. 2(d)), the pattern is relatively similar to that already seen for $R a=1 \times 10^{6}$. However, a very narrow stemmed, almost horizontal plume extends from the top left hand corner of the object to the left hand wall. Below the stem of the plume the fluid occupying the lower section of the cavity has a relatively high temperature (that decreases gradually towards the left hand while still remaining relatively high). This indicates that the peculiar circulation pattern established inside the fluid domain for this value of the Rayleigh number tends to create a heat island in its entire lower half. The heat released in the fluid by the vertical heated wall of the step cannot be transferred to the upper part of the cavity and tends to accumulate in the lower part causing an increase in the local temperature. The situation is clearly different in the upper domain where the heat released in the fluid by the horizontal heated wall can convectively be transferred to the top (cold) wall where it is extracted from the cavity. The frequency spectrum for this case (not shown), however, confirms that the flow is still time-periodic (the reader being referred to Sect. 3.1.4 for a discussion of the case $R a=1 \times 10^{8}$ ).

The values of the Nusselt number corresponding to all these situations can be found in Table III (where it is shown that the intensity of heat exchange between the surface of the step and the fluid increases as the Rayleigh number becomes higher). Interestingly, the set of values reported there also indicates that the mechanism of the Hadley type (being operative near the vertical side of the step) is much more efficient in transporting heat than that due to a heating-from-below condition at work along the horizontal side of the step (this finding being in line with other studies where square cavities with a heated plate built in vertically or horizontally or with a finned heated plate were considered, see, e.g., Oztop et al. [58]; Saravanana et al. [59]; Saravanan and Sivaraj [60]; Nadjib et al. [61]; we will come back to this concept in Sect. 3.1.3). 


\subsubsection{Patterning Behavior for the cavity with isothermal (hot) floor}

Having completed a sketch of the different situations which are established inside the cavity with the step for the case with adiabatic bottom wall, we now turn to examining the other fundamental situation with the bottom wall kept at constant temperature (heated wall, see Table IV). Most of these cases are summarized in Fig. 4.

For $R a \leq 1 \times 10^{5}$, the flow field descriptions already given for the adiabatic cases are more or less applicable to the hot floor cases. Appreciable differences, however, can be identified in the temperature field. In the hot floor case (see the right frames of Fig. 4), a relatively high temperature region is established where the floor of the cavity meets the vertical side of the step. In practice, a continuous hot boundary layer develops along all the heated surfaces. It consists of two horizontal branches and one vertical branch. A cold boundary layer can also be seen at the ceiling of the cavity. Increasing the Rayleigh number to $R a=1 \times 10^{5}$ (Fig. 4(b)) leads to a decrease in the thickness of the temperature boundary layers at the ceiling and the floor of the cavity and at the side and top of the step. Moreover, for this value of Ra (Fig. 4(b)) a recognizable plume begins to form at the top left hand corner of the step (protruding towards the top left hand corner of the cavity).

Table IV: Cases examined for the case of pure buoyancy convection with hot bottom wall.

\begin{tabular}{ccccc}
\hline$R a$ & Mesh Size & $N u_{\text {step }}^{\text {horiz }}$ & $N u_{\text {step }}^{\text {vert }}$ & Regime \\
\hline \hline $10^{3}$ & $40 \times 40$ & $\cong 1$ & 2.41656 & Steady \\
$10^{4}$ & $40 \times 40$ & 1.279432 & 2.0596 & Steady \\
$10^{5}$ & $80 \times 80$ & 3.12286 & 5.49028 & Steady \\
$10^{6}$ & $150 \times 150$ & 4.2792 & 9.72496 & Steady \\
$10^{7}$ & $270 \times 270$ & 6.05596 & 15.14134 & Steady \\
$10^{8}$ & $500 \times 500$ & $\cong 12.25$ (average) & $\cong 25.53$ (average) & Weakly Turbulent \\
\hline \hline
\end{tabular}

Though these patterns, with the exception of the additional branch of thermal boundary layer formed along the bottom wall, may be considered relatively similar to those already described for the other boundary condition, however, distinguishing features can be highlighted for $R a=1 \times 10^{6}$ in terms of structure of the flow field (Fig. 4(c)). Two almost symmetrical counter-rotating circular shaped rolls develop in the upper half of the cavity. At the same time, two other circular shaped velocity rolls develop in the lower half of the cavity. A very complex multi-cellular flow emerges with the maximum velocity magnitude being attained where the rolls meet in the upper half of the cavity. 
Accepted for publication in Int. J. Thermal Science, (ISSN: 1290-0729) on 11 May 2020
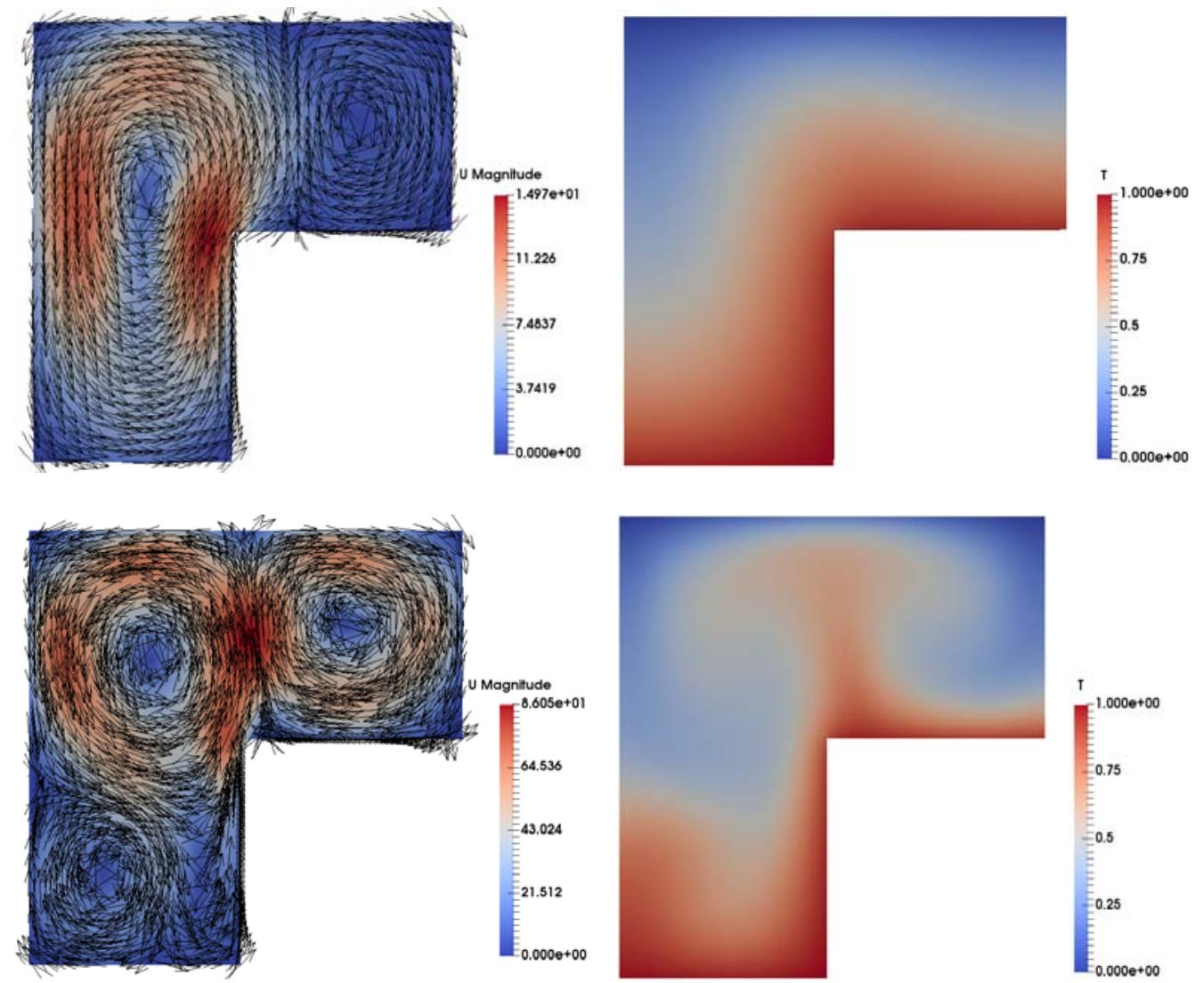

(a)
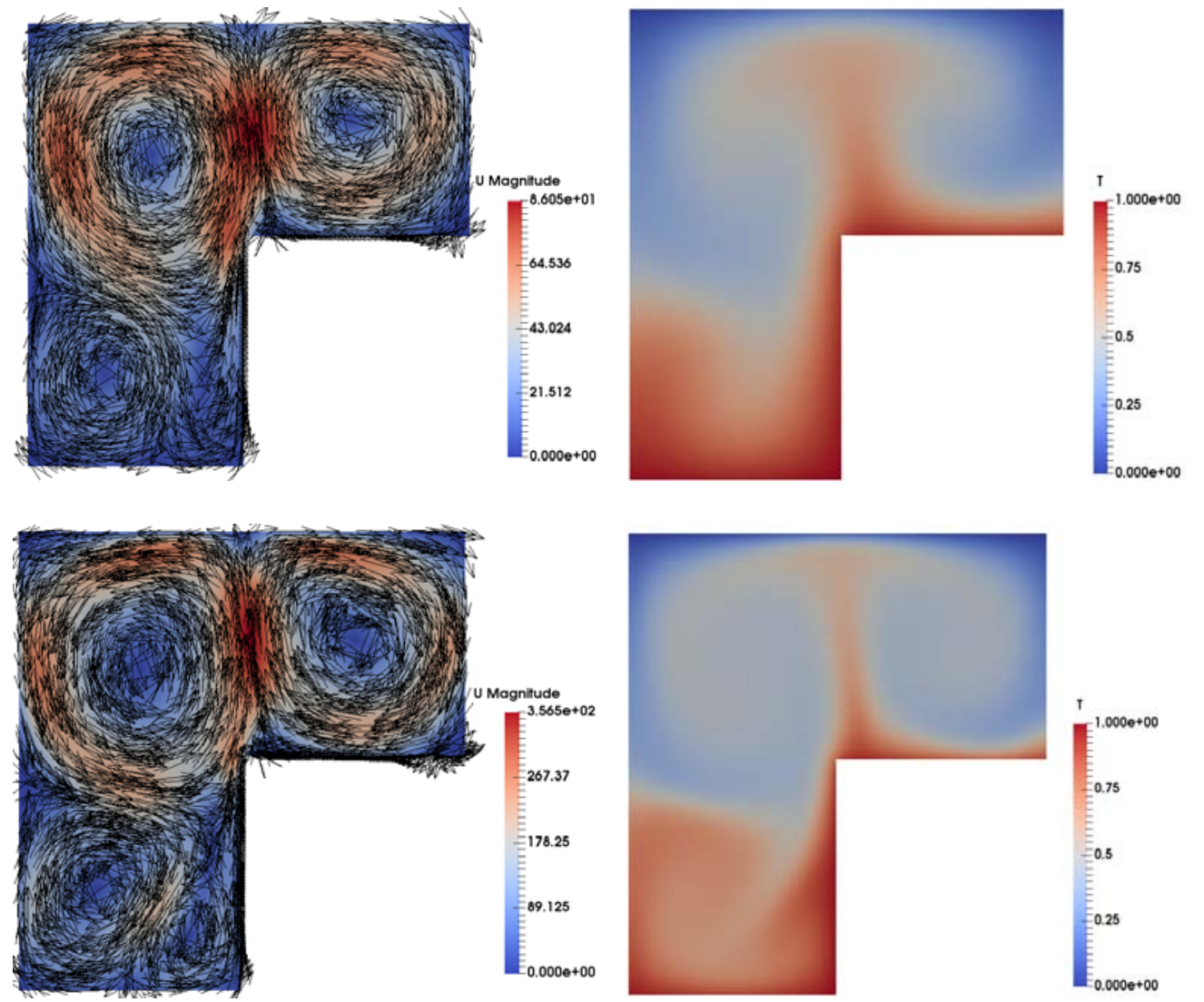

(b)
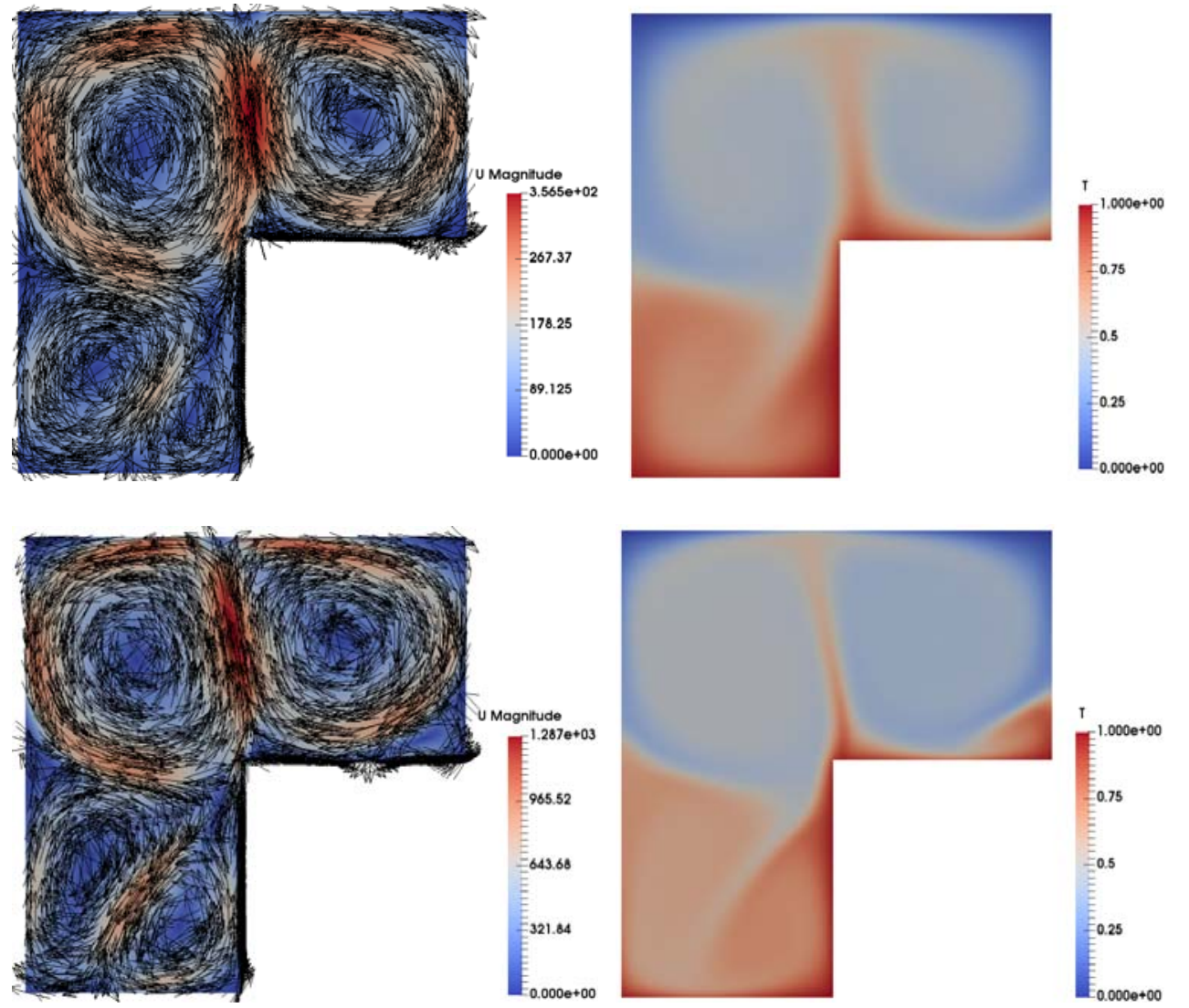

(c)

Figure 4: Snapshots of velocity field (left) and temperature distribution (right) for the case of pure buoyancy convection and cavity with hot bottom wall: a) $R a=10^{4}$, b) $R a=10^{5}$, c) $R a=10^{6}$, d) $R a=10^{7}$. 
Apart from the notable departure from the corresponding situation shown in Fig. 2(c) in terms of flow structure, other remarkable modifications concern the temperature field. The thermal plume, which in the case of adiabatic bottom was displaying an essentially horizontal direction (Fig. 2(c)), appears now with a main direction rotated by 90 degrees, i.e. it has an almost vertical structure (Fig. 4(c)). Moreover, the flow is steady (it was time-dependent for the case with adiabatic bottom).

When the Rayleigh number is increased to $R a=1 \times 10^{7}$ (Fig. 4(d)), most surprisingly, the flow is still steady. Four velocity rolls are developed like those seen for $R a=1 \times 10^{6}$.

For all these cases, interestingly, a second rising hot plume is established along the left wall of the cavity (not present when the bottom wall is adiabatic).

\subsubsection{Heat Exchange and related trends}

Notably, direct comparison of the information reported in Tables III and IV can be used to get interesting insights into the impact exerted by the different thermal boundary condition assumed for the bottom floor on the emerging dynamics. Indeed, it can be seen that the mechanisms of heat exchange being operative along the sides of the step sensitively depends on the type of bottom floor. The magnitude of heat exchange along the vertical side of the obstruction tends to decrease when the bottom floor is heated, which is a relatively counter-intuitive finding.

In practice, an explanation for this trend can be elaborated in its simplest form on the basis of the argument that the magnitude of heat removal largely depends on the specific structure of the velocity field, and that this structure is more favorable to heat exchange when the bottom floor is adiabatic (as in this case a significant amount of fluid can be transported towards the upper side of the step due to the existence of a single roll occupying the entire lower half of the cavity).

In such a context, it is also worth highlighting that $N u_{\text {step }}^{\text {vert }}$ for the cavity with the adiabatic floor (Table III) follows almost exactly the scaling law for the classical Hadley flow in an unobstructed square cavity, i.e. $N u \cong 0.13 \times R a^{0.31}$ (see, e.g., Yu et al. [62]; Corvaro and Paroncini [63]), which is in line with the considerations elaborated above about the presence of a single roll on the left of the vertical hot side of the step (resembling the classical Hadley flow which would be established in a classical square cavity with no step inside). As reported in Table IV, $N u_{\text {step }}^{\text {vert }}$ takes slightly smaller values when the hot floor is considered (with respect to the adiabatic case) for relatively high values of $R a$, which we ascribe to the aforementioned presence of a secondary roll developing in the lower half of the cavity (able to cause a decrease in the amount of fluid being transported upwards along the vertical face of the step).

Another outcome of the focused comparison of Table III and IV is the realization that regardless of the thermal condition implemented for the bottom floor, $N u_{\text {step }}^{\text {horiz }}$ is always smaller than $N u_{\text {step }}^{\text {vert }}$. This trend is relatively counterintuitive if one considers that in the light of existing studies for the pure Rayleigh-Bénard flow (see, e.g., Stevens et al. [64]), the Nusselt number should take values much higher than those reported in these tables and display $\mathrm{a} \cong R a^{\gamma}$ scaling with $2 / 7<\gamma<1 / 3$ ). 
The key to understanding these results lies in considering that the region located above the step (though it is heated form below and cooled from above) does not behave as a pure RB system. This is clearly witnessed by the lack of small scale thermal plumes, which one would expect for $R a \geq \mathrm{O}\left(10^{6}\right)$. Even for $R a=10^{7}$ no small scale plumes can be seen in the present case, see Figs. 4. By contrast, for pure RB flow and high values of $R a$, the flow is known to display the pervasive presence of small-scale plumes, continuously produced at the heated or cooled boundaries, which can later detach from such boundaries and have a two-fold remarkable effect: 1) contribute to increase the Nusselt number at the walls (see, e.g., Stevens et al. [64]), 2) support the overall erratic dynamics in the bulk of the fluid (see, e.g., Lappa [57]). As shown by the present results, such a mechanism is suppressed in the presence of an extended (hot) vertical surface in favor of large scale plumes originating from such surface or related hot corners.

\subsubsection{The progression towards Chaos}

Another (separated) discussion is needed for the values of the Rayleigh number required to produce a transition from the steady solution to a time-periodic (single frequency) state for the two fundamental situations corresponding adiabatic and isothermal bottom wall.

According to the results for the adiabatic floor case obtained by parametrically varying the Rayleigh number (Table III, Sect. 3.1.1), a Hopf bifurcation i.e. transition from stationary to oscillatory conditions should obviously take place somewhere in the range between $R a=10^{5}$ and $R a=10^{6}$.

We carried out dedicated simulations to identify precisely such a value and found it to be $R a_{\text {critical }} \cong 6.1 \times 10^{5}$.

For the hot floor case (Sect. 3.1.2, Table IV)), as the reader might have already realized at this stage, the transition is delayed to higher values of $R a$ (between $R a=10^{7}$ and $R a=10^{8}$, which is yet a relatively counter-intuitive fact if one considers that the flow should be more energetic in these conditions given the additional source of buoyancy represented by the portion of bottom wall kept at constant (hot) temperature). We found for this case $R a_{\text {critical }} \cong 1.1 \times 10^{7}$.

An interpretation of such counter-intuitive findings is not as straightforward as one would assume. A relevant explanation, however, can yet be rooted in the different nature and behavior of buoyancy flow according to the direction of the prevailing temperature gradient that generates it and the nature of the disturbances responsible for the first Hopf bifurcation, i.e. the transition from steady state to oscillatory flow.

These flows can be very different in terms of structure and undergo a completely different hierarchy of bifurcations. As an example, while the critical Rayleigh number for the transition to oscillatory flow of classical RB convection in a square cavity heated from below, cooled from above and with vertical adiabatic walls is $\mathrm{O}\left(10^{5}\right)$ in the case of air (Goldhirsch et al. [65]; Bouabdallah et al. [66]), the corresponding value for the case in which the cavity is rotated by $90^{\circ}$ (differentially heated vertical walls and adiabatic horizontal boundaries) is $\mathrm{O}\left(10^{8}\right)$ (Paolucci and Chenoweth [67]; Le Quéré and Behnia [68]). As also shown by other authors for the companion case of inclined systems 
(see, e.g., [54]), when vertical and horizontal temperature gradients are present at the same time (as in the present case), the above-mentioned two types of convection (Hadley \& RB) can interact in a relatively complex way and cause flow stabilization or destabilization depending on the prevailing effect.

In the light of all these arguments, further insights into the scenario revealed by the present numerical simulations are yielded in this section on the basis of a direct analysis of the flow disturbances (these disturbances have been obtained by subtracting the time-averaged velocity field to the instantaneous velocity).
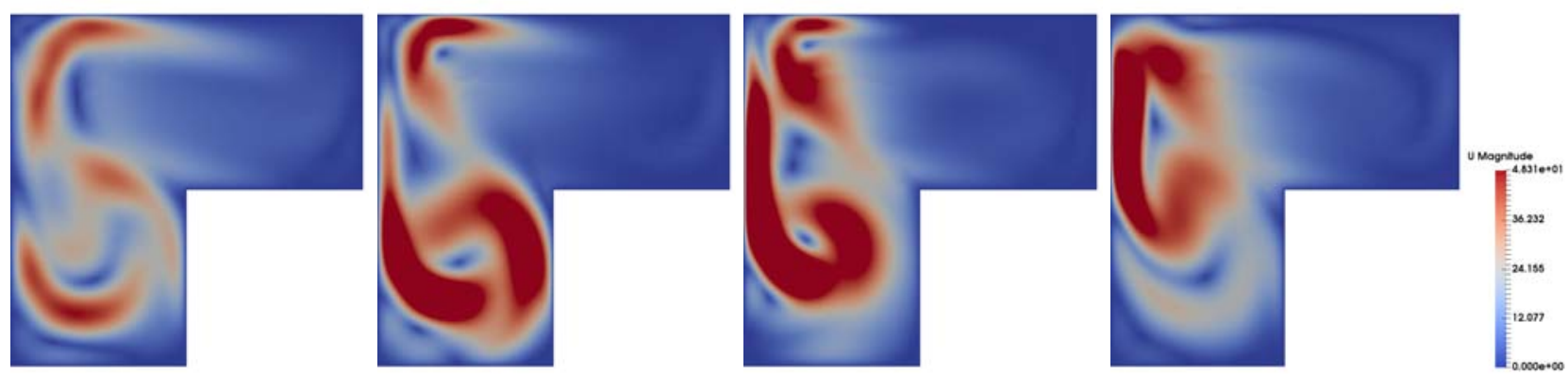

Figure 5: Four snapshots of velocity disturbances evenly spaced in time for the cavity with adiabatic floor, $R a=1 \times 10^{6}$.

Notably, in Fig. 5 it can be seen that for the adiabatic floor case, disturbances behave as a spiraling wave travelling in the clockwise direction along the boundary including the left side of the step, the bottom, floor and the entire left sidewall of the cavity. Following a cyclic process, a velocity peak originates in proximity to the corner of the step, it is then transferred to the lower roll that transports it from the left side of the step to the left side of the cavity. The disturbance then rises along the left wall until it reaches the left top corner where it is damped (giving rise to a new cycle).

As already reported in Sect 3.1.1, increasing the Rayleigh number to $10^{7}$ does not substantially modify this mechanism. For $R a=1 \times 10^{8}$, however, a notable change in the temporal behavior occurs. This is shown in Fig. 6. For this value of $R a$, a heat island located in the lower half of the cavity is still a characteristic of the temperature pattern (Fig. 6(a)). However, as witnessed by Fig. 6(b), the frequency spectrum becomes much more complex (e.g., with respect to that seen in Fig. 3). It now displays a continuous interval of frequencies.
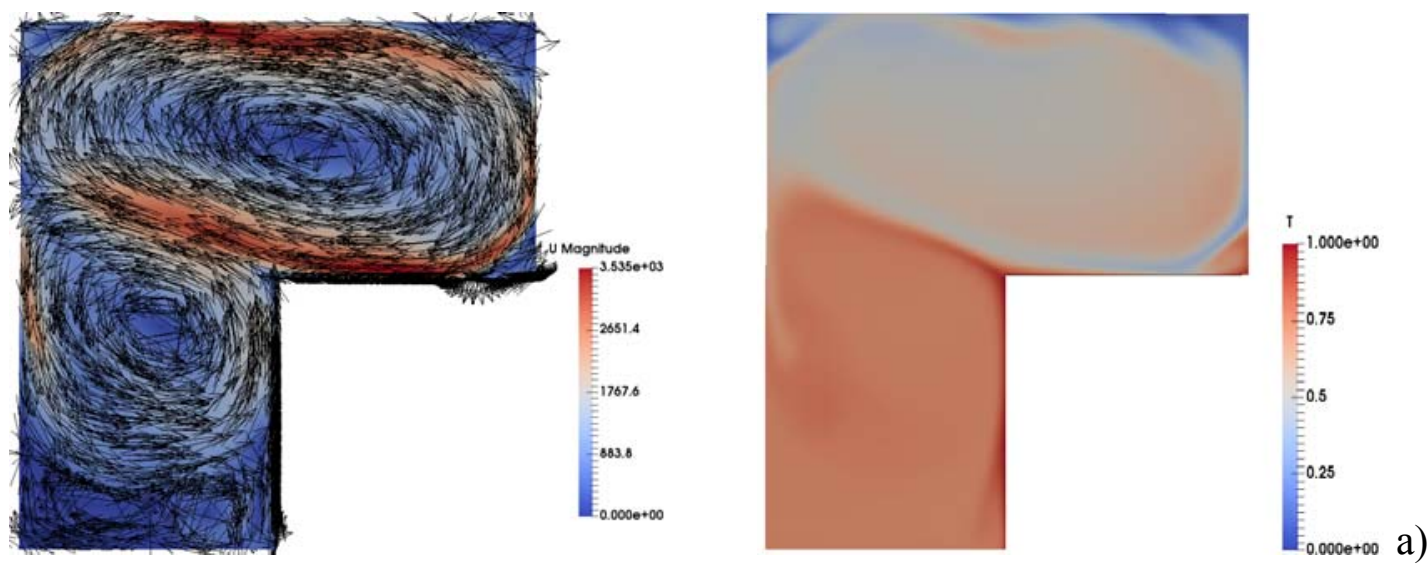


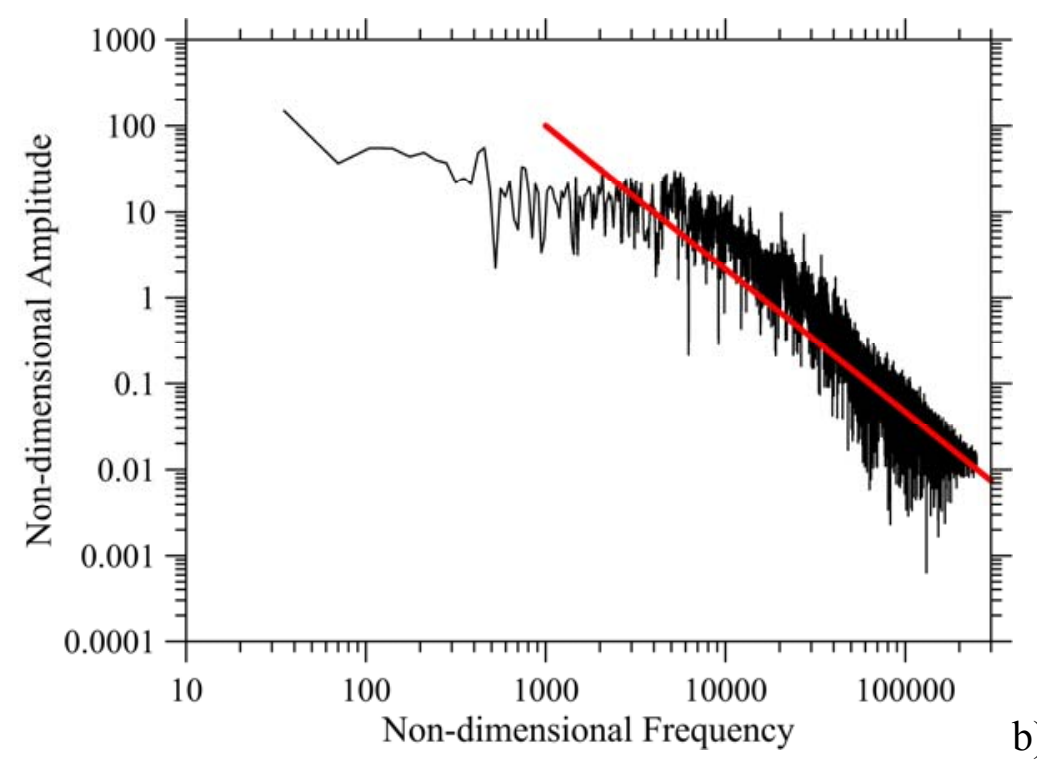

Figure 6: Pure buoyancy convection in cavity with adiabatic bottom wall, $R a=1 \times 10^{8}$ : (a) snapshot of velocity field (left) and temperature distribution (right), (b) frequency spectrum related to the velocity signal measured by a numerical probe located at $\mathrm{x}=0.25$ and $\mathrm{y}=0.75$ (the red line indicates the Kolmogorov scaling law).

Interestingly, it can also be seen that the frequency spectrum (frequency and related amplitude plotted using logarithmic scales for the axes) aligns to a good approximation with a $\omega^{-5 / 3}$ law in a certain range of frequencies (from $\mathrm{O}\left(10^{3}\right)$ to $\mathrm{O}\left(10^{5}\right)$, the reader being referred to the solid red line in Fig. 6(b)).

The key to understand these results lies in considering the well-accepted idea that, under certain length scales, fluid flow starts to behave following a universal (repetitive) behavior. This physical intuition, originally elaborated by Kolmogorov [69-72], is nowadays known as the inertial turbulence theory; the related interval where the energy spectrum aligns with a $-5 / 3$ law is the socalled inertial range of space scales (where turbulence is homogeneous and isotropic $[51,73]$ ).

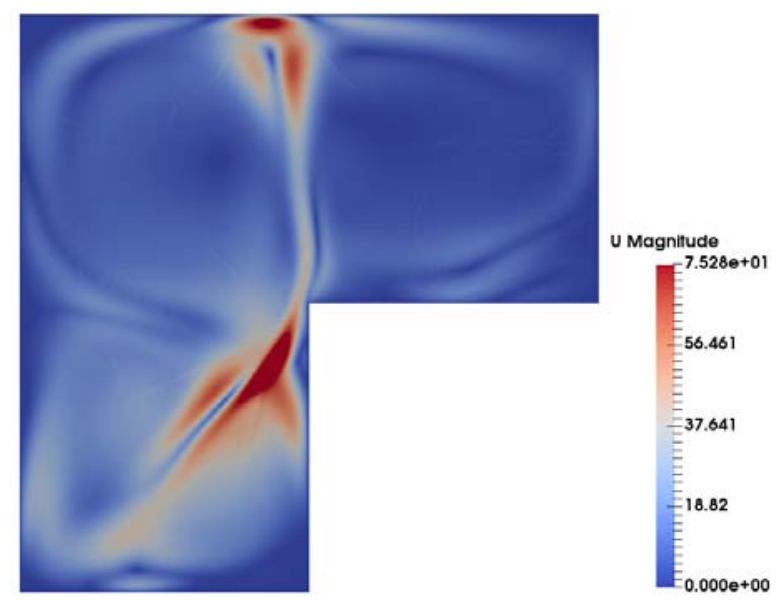

Figure 7: Snapshot of the velocity disturbances for the cavity with the hot floor, $R a=1.15 \times 10^{7}$. 
The bifurcation scenario dramatically changes when the configuration with the hot bottom is considered. As shown in Fig. 7 for slightly supercritical conditions ( $R a$ slightly larger than $R a_{\text {critical }} \cong 1.1 \times 10^{7}$ ), the disturbances do not behave as a wave; rather they tend to be localized in the stem and cap of the vertical hot plume originating from the corner of the step and in the inclined descending plume of cold fluid (corresponding to the line of contact between the two rolls located in the lower part of the domain). While for the adiabatic-floor case (Fig. 5) a kinship may be identified with the typical disturbances known to affect the classical Hadley flow in unobstructed cavities (generally taking the form of waves travelling along the solid boundary, see, e.g., [68]), this case should be regarded as a realization of the typical instabilities that can affect plumes in the IVND regime [55, 74-77]. These instabilities are generally induced by the horizontal shear in the bulk of the fluid (which can take relatively high values for the conditions considered in the present work, especially in proximity to the stem of the plumes originating from the corner of the step).

Put simply, Fig. 7 reveals that the mechanism for the transition from steady to time-dependent conditions can sensitively depend on the type of thermal conditions considered for the bottom floor. Indeed, the main cause of the bifurcation is transferred from the boundary-layer mechanism seen in Fig. 5 for the adiabatic floor to the thermal-plume driven one for the situation with the isothermal hot floor.

As a concluding remark for this section, we wish to highlight that also for this boundary condition, the frequency spectrum can be made more involved on increasing further $R a$ (as illustrated in Fig. 8).

As the reader will realize by inspecting this figure, some differences can be noticed with respect to Fig. 6 (owing to the different mechanisms responsible for the excitation of oscillatory flow, the spectrum is more energetic). Nevertheless, the amplitudes still align to a good approximation with the aforementioned Kolmogorov law over a limited range of frequencies, representative of the universal behavior taken by turbulence on the small scales [54].
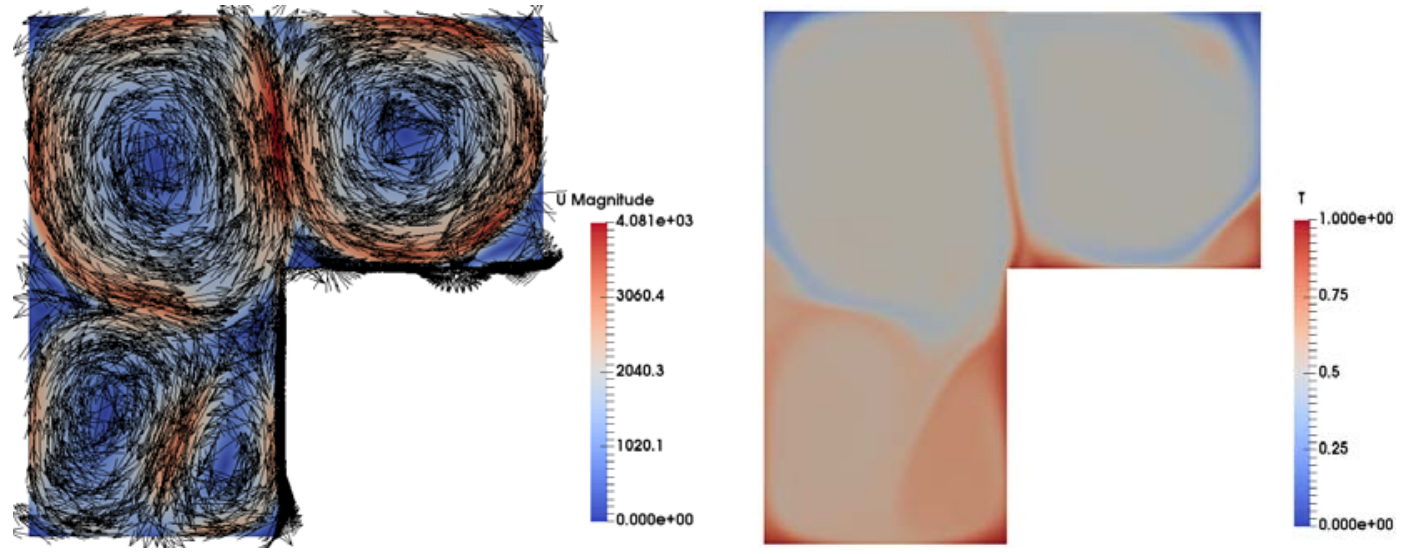


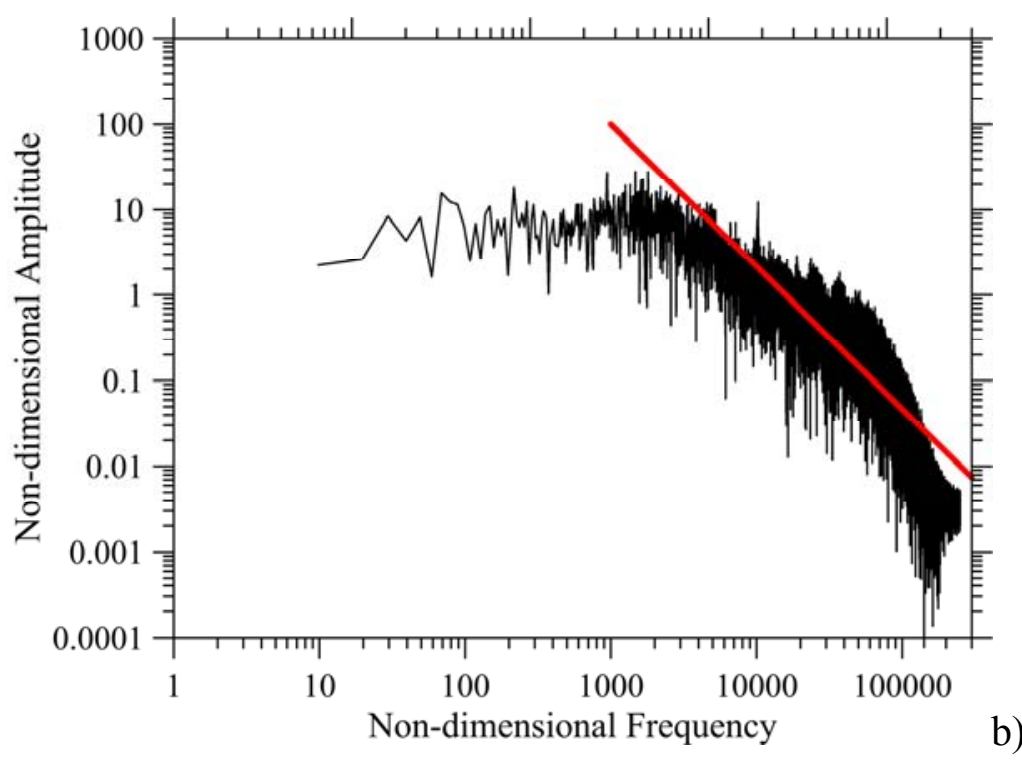

Figure 8: Pure buoyancy convection in cavity with hot bottom wall, $R a=1 \times 10^{8}$ : (a) snapshot of velocity field (left) and temperature distribution (right), (b) frequency spectrum related to the velocity signal measured by a numerical probe located at $\mathrm{x}=0.25$ and $\mathrm{y}=0.75$ (the red line indicates the Kolmogorov scaling law).

\subsection{Cavity with coaxial inflow and outflow sections}

The foregoing discussion has deliberately been limited to illustrating the dynamics for pure buoyancy convection. The present section continues this inquiry by probing the additional role played by forced convection (due to cold fluid being injected through an inflow section located on the left sidewall). Our specific aim in this regard is to assess the changes experienced by convection, its route to chaos and related patterning scenario in the presence of forced flow over the same range of values of the Rayleigh number considered in the preceding section for a fixed value of the Richardson number (corresponding to buoyant and forced flow having a comparable strength or intensity), i.e. $R i=1$. For the purpose of quantifying these effects, we stick to the same two alternate configurations already examined in Sect. 3.1, i.e. the case in which the bottom wall of the cavity is adiabatic or kept at a fixed temperature.

We could spot several interesting effects in term of the behavior of the flow and temperature distribution. For consistency with the approach already undertaken in Sect. 3.1, first we describe the numerical simulations conducted for the adiabatic floor and then we move on to the second case. 
Accepted for publication in Int. J. Thermal Science, (ISSN: 1290-0729) on 11 May 2020
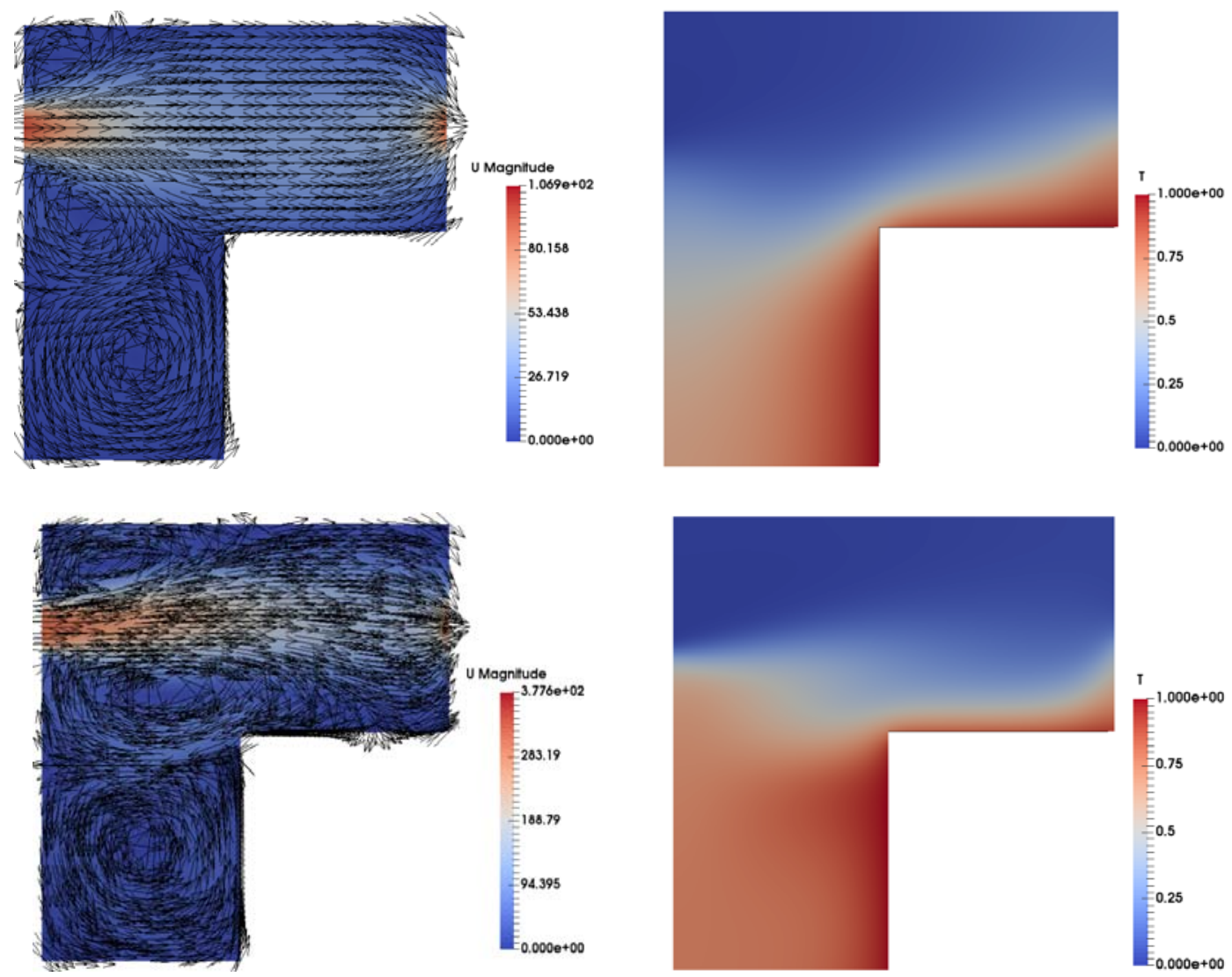

(a)
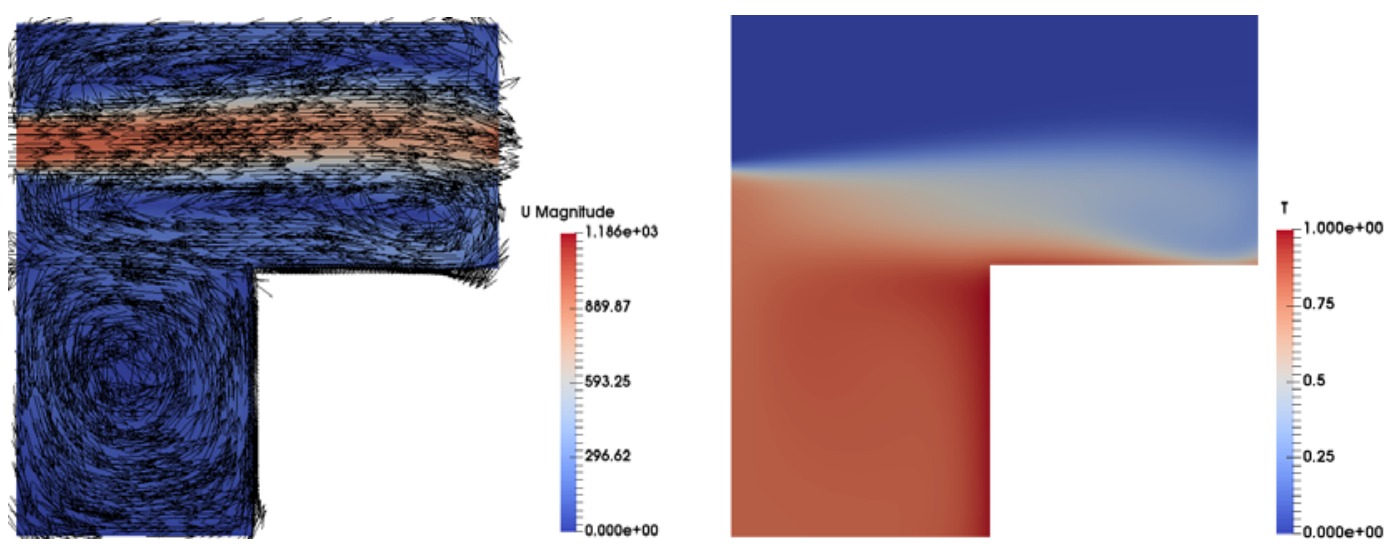

(b)
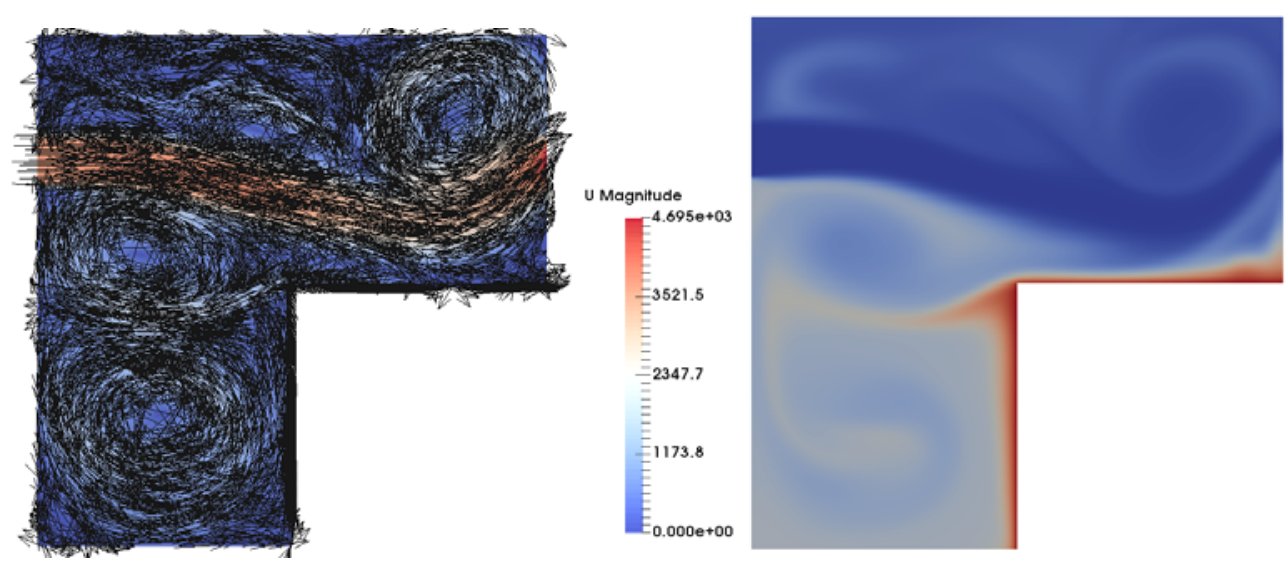

(c)

Figure 9: Snapshots of velocity field (left) and temperature distribution (right) for the case of hybrid forced/buoyancy convection $(R i=1)$, coaxial inflow and outflow sections and cavity with adiabatic bottom wall: a) $R a=10^{4}$, b) $R a=10^{5}$, c) $R a=10^{6}$, d) $R a=10^{7}$. 
Table V: Cases examined for hybrid forced/buoyancy convection $(R i=1)$, coaxial inflow and outflow sections and cavity with adiabatic bottom wall.

\begin{tabular}{ccccc}
\hline \hline$R a$ & Mesh Size & $N u_{\text {step }}^{\text {horiz }}$ & $N u_{\text {step }}^{\text {vert }}$ & Regime \\
\hline \hline $10^{4}$ & $40 \times 40$ & 2.69812 & 4.88732 & Steady \\
$10^{5}$ & $80 \times 80$ & 2.08836 & 7.02482 & Steady \\
$10^{6}$ & $120 \times 120$ & 2.1104 & 13.18764 & Steady \\
$10^{7}$ & $270 \times 270$ & $\cong 16.7$ (average) & $\cong 34.2$ (average) & Moderately \\
& & & & Turbulent \\
\hline \hline
\end{tabular}

Following a logical process, with systems of increasing complexity being described as the discussion progresses, we begin our analysis from the simplest case, namely, that shown in Fig. 9(a) $\left(R a=1 \times 10^{4}\right)$. One velocity roll, mainly of buoyancy nature is formed in the lower portion of the cavity (oriented in the counter-clockwise sense in the figure). It can also be seen that the stream (jet) of cold fluid entering through the inlet spreads itself in the vertical direction and forms a nozzle-like structure in the upper part of the cavity (Fig. 9(a)). This structure is maintained up to the outlet. In proximity to the inflow section, a secondary (minor) roll develops between this current and the aforementioned main roll of buoyancy nature. The fluid attains its highest velocity at the inlet and the outlet. Moreover, relatively high temperature regions are located in proximity to the vertical and horizontal wall of the step. The flow is steady.

For $R a=1 \times 10^{5}$ (Fig. 9(b)), the velocity field is more complex. An increase in $R a$ and Re at fixed $R i$ obviously causes a rise of the magnitude of velocity. Interestingly, two main rolls exist in this situation. The first roll (square shaped) has a larger extension and it is located in the bottom portion of the cavity (yet we argue it is essentially of buoyancy nature as the fluid rises along the heated wall of the step). The second roll, relatively smaller, is located just above the mid-plane of the cavity and it is slightly elongated in the horizontal direction. It can be noticed that the 'nozzle-like' streamline structure that was formed for $R a=1 \times 10^{4}$ is disturbed in this case owing to the formation of this roll (which tends to alter the flow directed form left to right and to prevent it from spreading freely in the vertical direction). The fluid enters from inlet with a high velocity, it continues to travel straight and then starts to spread after it has passed the middle portion of the cavity. In Fig. 9(b) it is also evident that the heat is accumulated entirely in the lower portion of the cavity due to the amount of heat laterally released from the hot step and the scarce mixing occurring between the fluid located in the upper and lower portions of the cavity. This develops a heat island in the entire lower half of the cavity. This island partially protrudes into the upper portion of the cavity near the inflow section owing to the presence of the aforementioned second roll. Moreover, a relatively thick horizontal thermal boundary layer is formed above the hot step. The flow is steady.

Continuing with the description of the modifications induced by an increase in $R a$, for $R a=1 \times 10^{6}$ (Fig. 9(c)) the temperature distribution is very similar to the previous situation with a heat island 
still present in the entire lower half. In this case the horizontal return flow parallel to the main jet (warm fluid moving from left to right along the top surface of the step) also contributes to this effect. The flow is still steady.

On increasing the Rayleigh number to $R a=1 \times 10^{7}$ (Fig. 9(d)), however, a marked change is produced, i.e. the emergence of three equally sized velocity rolls. The first one is formed in the lower portion of the cavity as a result of buoyancy (fluid rising along the vertical wall of the step). Another one forms close to the ceiling in the upper portion of the cavity due to the combined effect of buoyancy and fluid flowing from the inflow section towards the outflow section. Remarkably, it causes a disruption in the straight movement of the fluid entering from the inlet. Notable changes can also be spotted in the case of temperature field (Fig. 9(d)). The heat island occupying the lower half in the previous cases disappears and only a hot thin boundary layer can be spotted along the vertical wall and a thicker one along the horizontal wall of the step. The flow is relatively turbulent in this case as shown by the frequency spectrum in Fig. 10. Interestingly, it aligns with the Kolmogorov law over a range more extended (from $\mathrm{O}\left(10^{3}\right)$ to $\mathrm{O}\left(10^{6}\right)$ ) than that seen for the corresponding configuration with no inflow and outflow sections (which explains why it is referred to as 'moderately turbulent' in Table V).

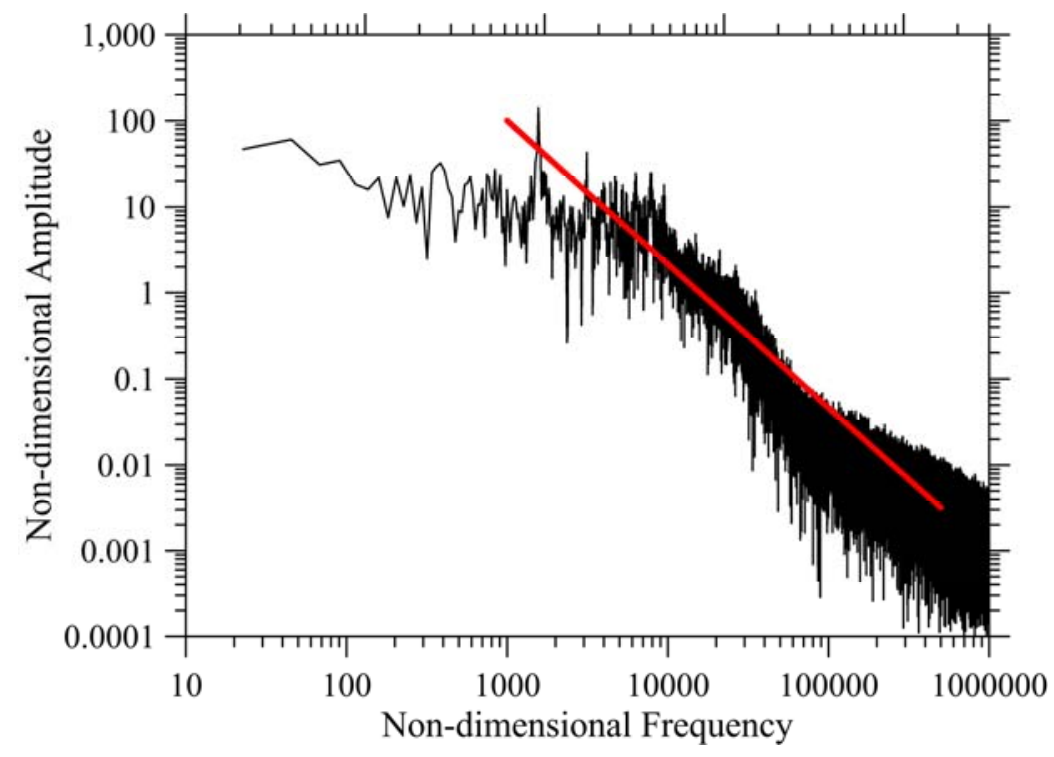

Figure 10: Hybrid convection in cavity with adiabatic bottom wall, $\mathrm{Ra}=1 \times 10^{7}$ : (a) frequency spectrum related to the velocity signal measured by a numerical probe located at $x=0.25$ and $y=0.75$ (the red line indicates the Kolmogorov scaling law).

Following up on the previous point about the suppression of the heat island effect, such change can be ascribed to the topological bifurcation displayed by the stream of cold fluid entering the cavity from the left. As shown in Fig. 9(d), owing to the presence of a new vortex (located just under the inflow section and driven by the joint effects of forced and buoyancy convection) such a stream breaks into two branches before reaching the step and the lower stream is routed into the lower domain, thereby strongly contributing to mitigate the temperature in that area. Moreover, it can be 
seen that, since the main horizontal jet originating from the inlet is distorted and impinges on the top surface of the obstruction, the aforementioned 'return flow' transporting heat from the entire top surface of the step directly into the left region is suppressed.

Notably, another way to somehow interpret all these results is to take a look at Table $\mathrm{V}$ and focus on the behavior of the Nusselt number. The most striking variation indeed occurs for $R a=1 \times 10^{7}$, for which a notable change can be seen in the relative importance of $N u_{\text {step }}^{\text {hori }}$ and $N u_{\text {step }}^{\text {vert }}\left(N u_{\text {step }}^{\text {horiz }}=15 \%\right.$ and $50 \%$ of $N u_{\text {step }}^{\text {vert }}$ for $R a=10^{6}$, and $R a=10^{7}$, respectively). In particular, the parameter $N u_{\text {step }}^{\text {horiz }}$ jumps from a value $\cong 2$ for $R a=10^{6}$ to $\cong 17$ for $R a=1 \times 10^{7}$.

An interpretation for this fascinating trend can easily be elaborated considering again the flow topological arguments provided above. A close look at Fig. 9(d), indeed reveals that what sets this specific value of the Rayleigh number apart from the other cases considered earlier is the fact that, after being split into two main branches (the lower one being funneled into the lower domain as explained before), the upper branch of cold fluid originating from the inflow section hits directly the top surface of the step thereby strongly contributing to the heat exchange there.

Cross comparison of Table V with Table III is also instructive as it shows (as expected) that replacing a top wall steadily maintained at a cold temperature with a limited amount of cold fluid being injected into the cavity per unit time at $R i \cong 1$, makes the heat removal process from the step horizontal wall less efficient for moderate values of $R a$ (obviously this observation only applies to the specific flow rate considered in the present work, additional studies being required in the future to parametrically investigate the effect of the non-dimensional size of the inflow and outflow sections).

We turn now to the other case in which the floor is kept at a constant temperature (Table VI). For $R a=1 \times 10^{4}$ (Fig. 11(a)) the flow pattern is almost the same already seen before with a slight difference in the structure of the rolls. It can be noticed in the velocity field that the size of the velocity roll close to the bottom is relatively smaller with respect to the case with adiabatic floor and it is unable to take a square like shape. To justify this behavior it is convenient to start from the simple remark that the buoyant effect due to heating from below now directly contributes to the secondary roll located in proximity to the inflow section. As a result, it tends to become larger and expand into the lower region of the cavity.

Table VI: Cases examined for hybrid forced/buoyancy convection $(R i=1)$, coaxial inflow and outflow sections and cavity with hot bottom wall.

\begin{tabular}{ccccc}
\hline \hline$R a$ & Mesh Size & \multicolumn{1}{c}{$N u_{\text {step }}^{\text {horiz }}$} & \multicolumn{1}{c}{$N u_{\text {step }}^{\text {vert }}$} & Regime \\
\hline \hline $10^{4}$ & $40 \times 40$ & 2.13828 & 4.96214 & Steady \\
$10^{5}$ & $80 \times 80$ & 1.289478 & 7.0278 & Steady \\
$10^{6}$ & $120 \times 120$ & 1.040622 & 13.22984 & Steady \\
$10^{7}$ & $270 \times 270$ & $\cong 13.5$ (average) & $\cong 38.9$ (average) & Moderately \\
& & & & Turbulent \\
\hline \hline
\end{tabular}


Accepted for publication in Int. J. Thermal Science, (ISSN: 1290-0729) on 11 May 2020
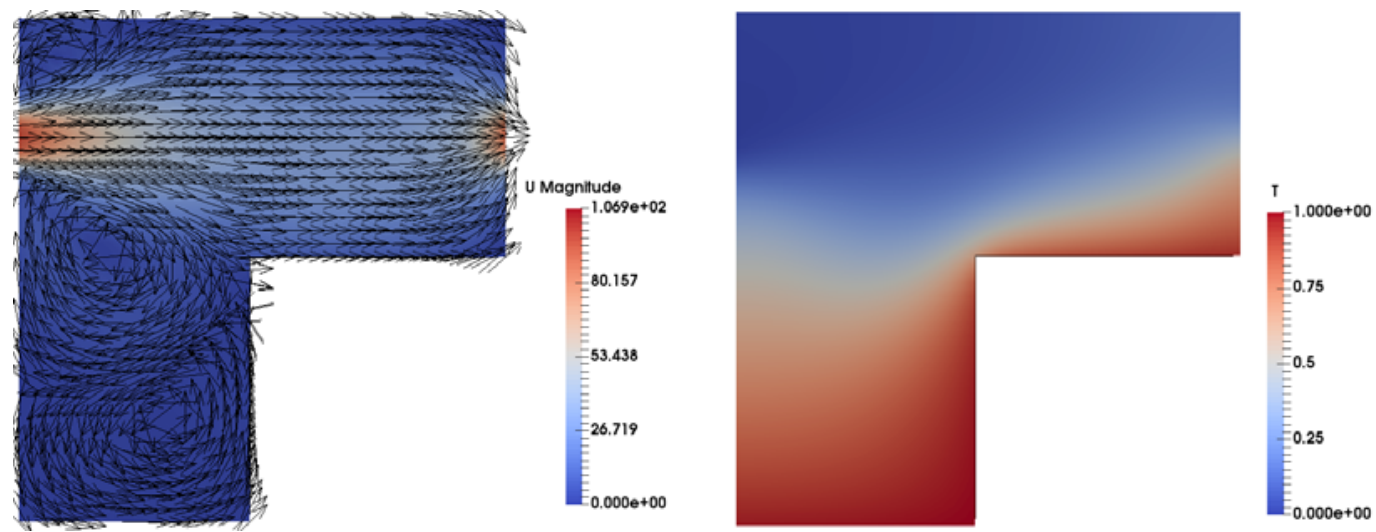

(a)
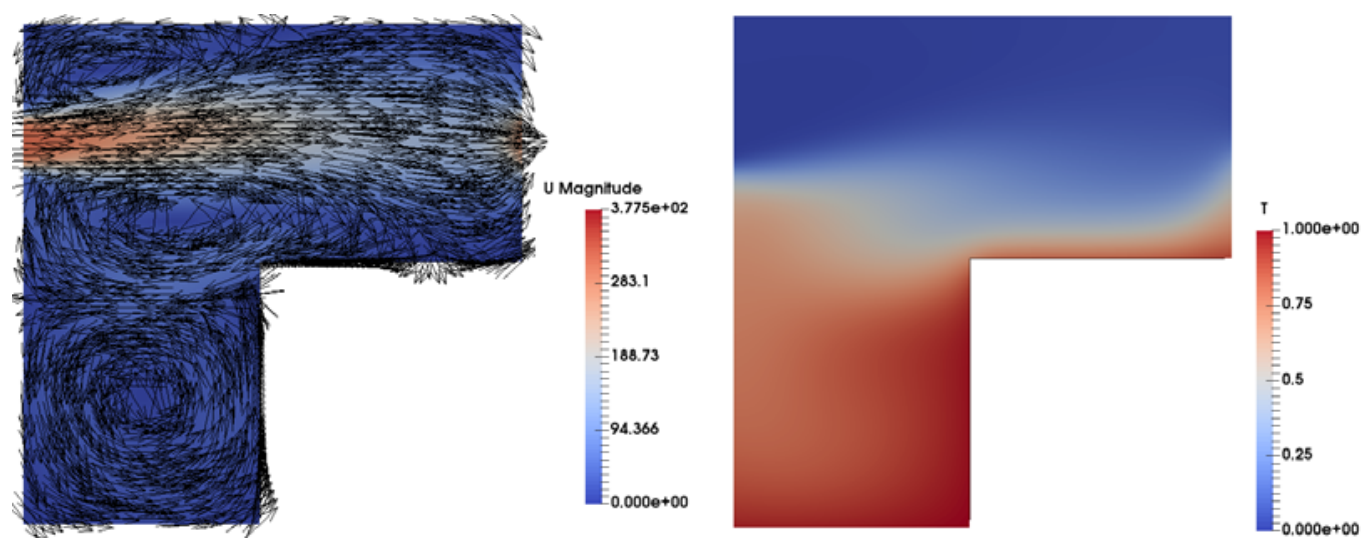

(b)
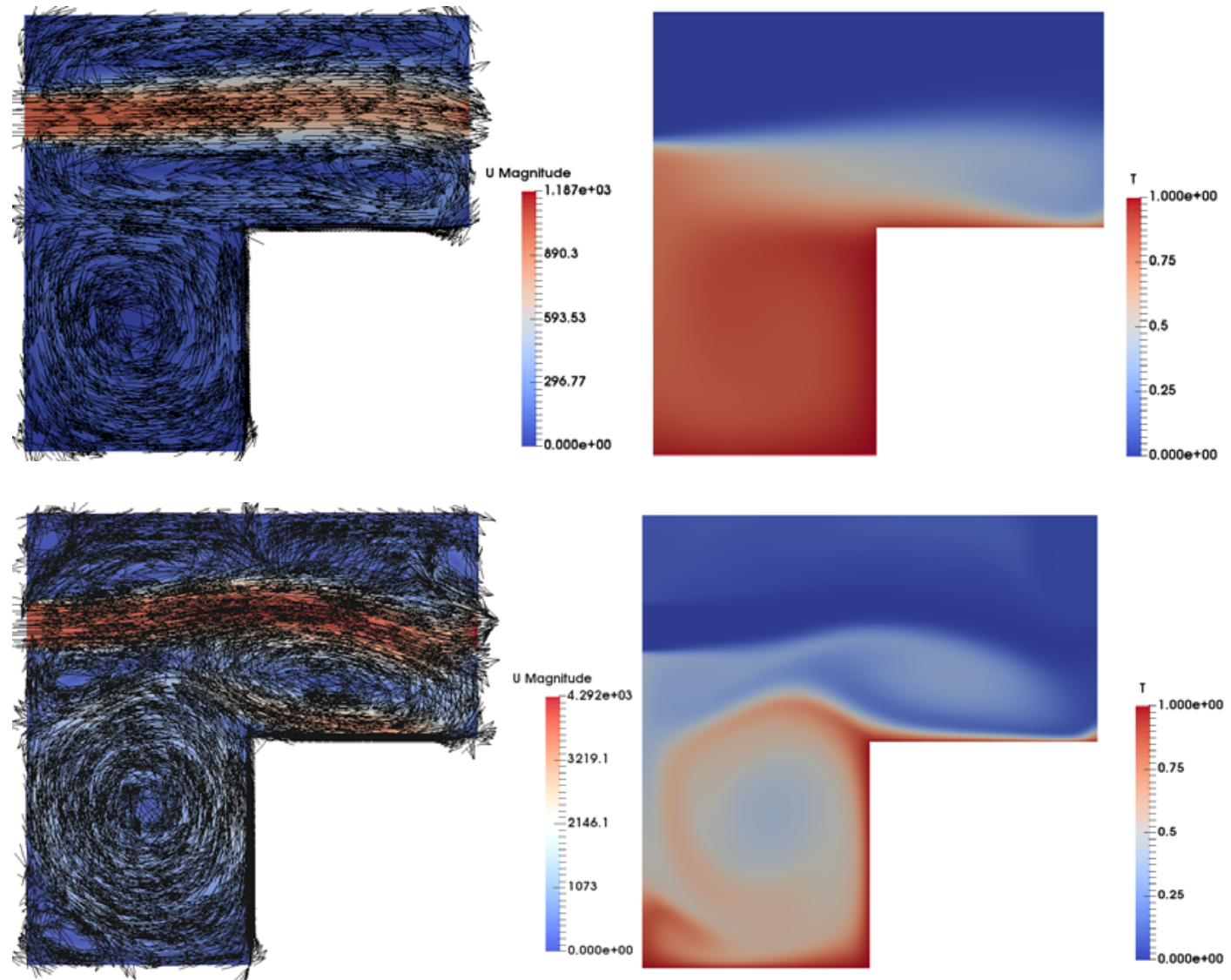

(c)

Figure 11: Snapshots of velocity field (left) and temperature distribution (right) for the case of hybrid forced/buoyancy convection $(R i=1)$, coaxial inflow and outflow sections and cavity with hot bottom wall: a) $R a=10^{4}$, b) $R a=10^{5}$, c) $R a=10^{6}$, d) $R a=10^{7}$. 
For $R a=1 \times 10^{5}$ and $R a=1 \times 10^{6}$ (Fig. 11(b) and (c)) no significant differences can be highlighted in terms of the flow and temperature patterns as they are roughly the same as for the adiabatic floor case.

Once again, a significant departure, however, can be seen when $R a=1 \times 10^{7}$ is considered (Fig. 11(d)). Though in this case, the heat released by the top surface of the step can reach the left region, the temperature in the lower portion is mitigated by the cold fluid entering the cavity. Indeed, part of this fluid is directly injected in the lower half due to the presence of a small eddy located in proximity to the inflow section (just under the main jet). Fluid motion is still moderately chaotic as witnessed by the time-velocity plot in Fig. 12.

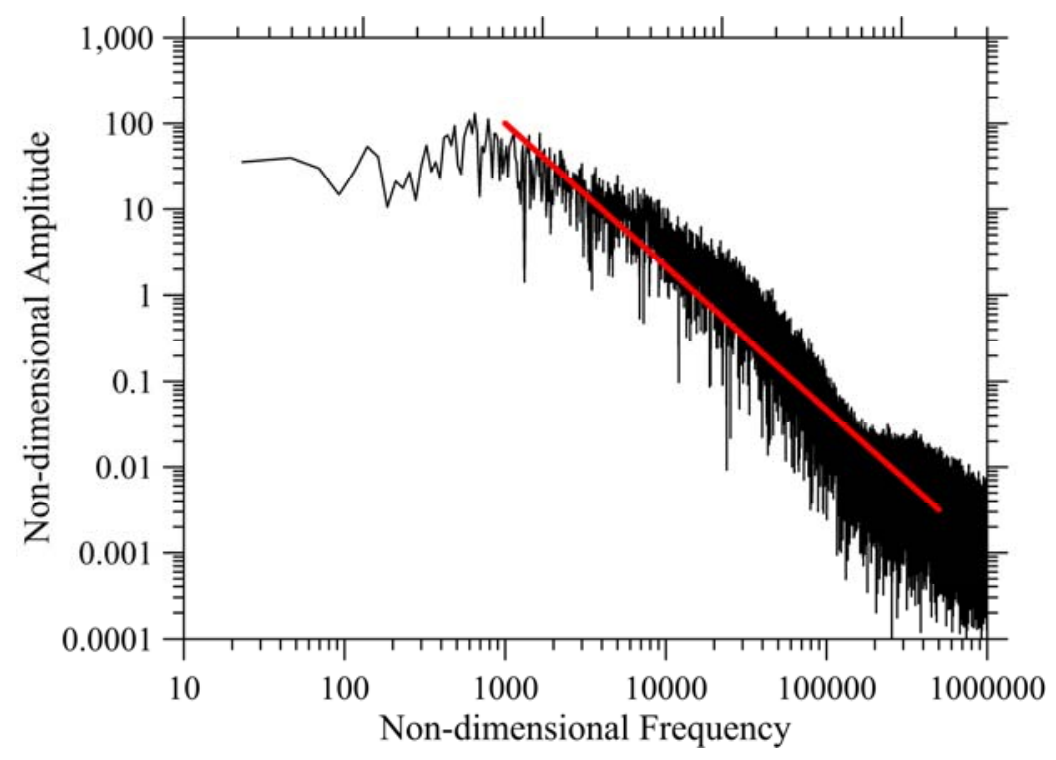

Figure 12: Hybrid convection in cavity with hot bottom wall, $R a=1 \times 10^{7}$ : (a) frequency spectrum related to the velocity signal measured by a numerical probe located at $\mathrm{x}=0.25$ and $\mathrm{y}=0.75$ (the red line indicates the Kolmogorov scaling law).

In order to clarify the evolution of these systems when the control parameter is increased, we have also evaluated precisely the critical values of the Rayleigh number for the transition from steady to time-periodic (single-frequency) flow. We found these to be $R a_{\text {critical }} \cong 1.2 \times 10^{6}$ for both cavities with adiabatic and hot bottom wall, respectively.

The related disturbances are reported in Fig. 13. This figure is instructive as it shows that thermal boundary layers or plumes are no longer at the root of the instability mechanism. As a comparison of Fig. 13a and 13b with Figs. 5 and 7 would immediately reveal, disturbances are now located essentially in the right portion of the cavity and there is strong evidence for their essentially vertical-shear-driven nature (the interested reader being also referred to the similar studies recently carried out by Ali and Dey [78] and Hemchandra et al. [79]). 

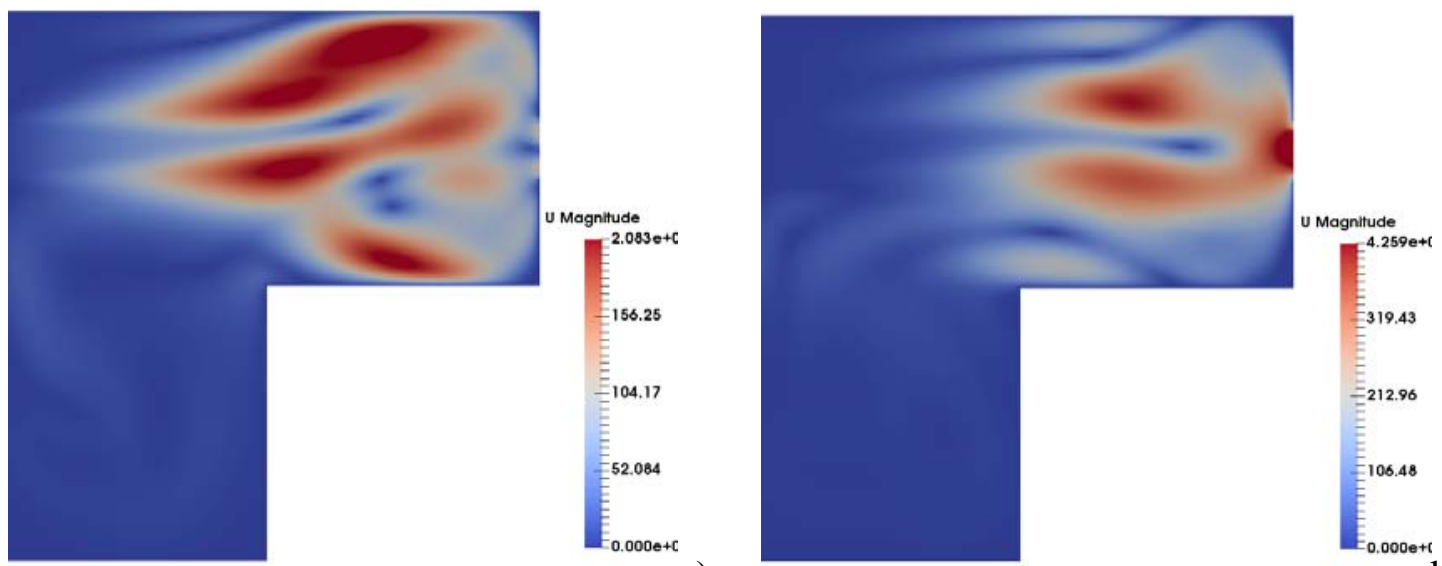

a)

b)

Figure 13: Snapshot of velocity disturbances for the case of hybrid forced/buoyancy convection $(R i=1)$, coaxial inflow and outflow sections: a) cavity with adiabatic floor, $R a \cong 1.25 \times 10^{6}$; b) cavity with hot floor, $R a=1.25 \times 10^{6}$

\subsection{Cavity with misaligned inflow and outflow sections}

Table VII: Cases examined for hybrid forced/buoyancy convection $(R i=1)$, misaligned inflow and outflow sections and cavity with adiabatic bottom wall.

\begin{tabular}{ccccc}
\hline \hline$R a$ & Mesh Size & $N u_{\text {step }}^{\text {horiz }}$ & $N u_{\text {step }}^{\text {vert }}$ & Regime \\
\hline \hline $1 \times 10^{4}$ & $40 \times 40$ & 6.93572 & 3.5079 & Steady \\
$1 \times 10^{5}$ & $80 \times 80$ & 12.09462 & 4.70892 & Steady \\
$1 \times 10^{6}$ & $120 \times 120$ & 21.0776 & 9.35676 & Steady \\
$1 \times 10^{7}$ & $270 \times 270$ & $\cong 40.3$ (average) & $\cong 31.6$ (average) & Weakly \\
& & & Turbulent \\
\hline \hline
\end{tabular}

After discussing in the previous section the dynamics of hybrid forced-buoyancy convection as a result of cold fluid injection through an inflow section located on top of the left sidewall, we finally address the case for which the inflow section is positioned relatively close to the floor. The relative height of the outflow section is not varied with respect to the previous analysis, i.e. it is located along the right wall in the upper part of the cavity (above the step at a certain distance from its horizontal surface).

Though this problem may be regarded as formally equivalent to that treated earlier, and the implemented change may appear as a minor variation, its effects can be dramatic, as further discussed in the remainder of this section (Figs. 14-17). 
Accepted for publication in Int. J. Thermal Science, (ISSN: 1290-0729) on 11 May 2020
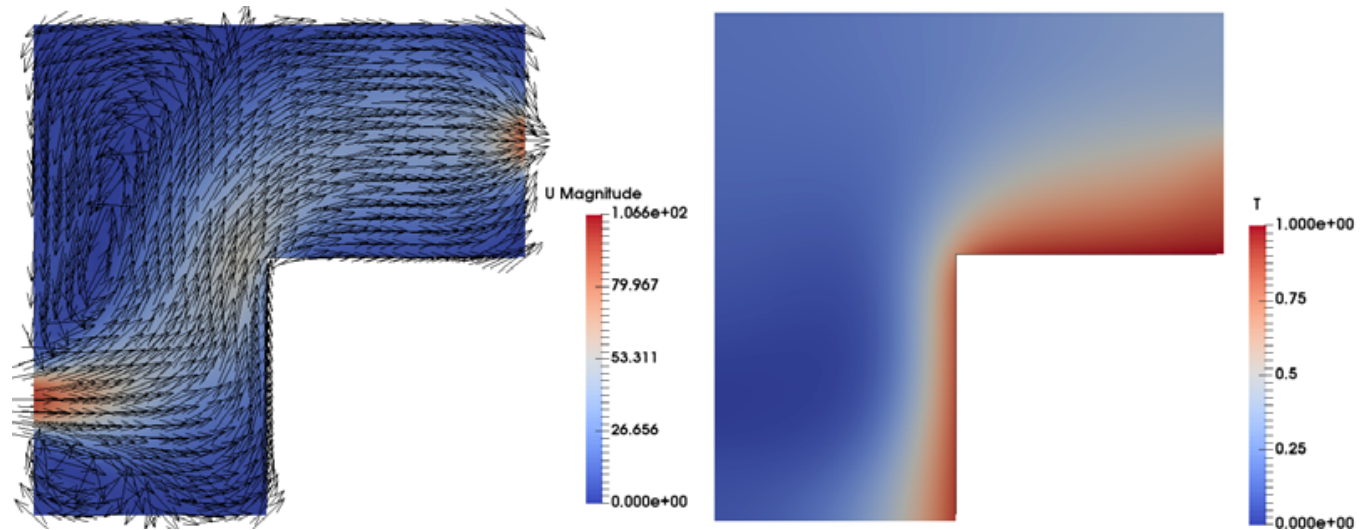

(a)
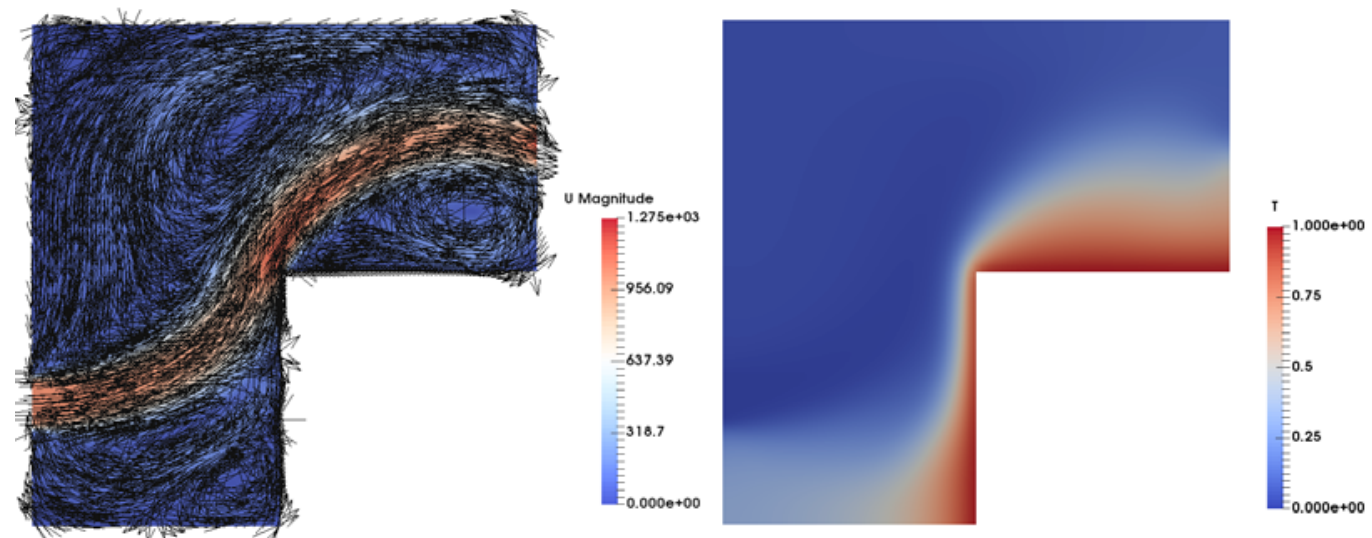

(b)
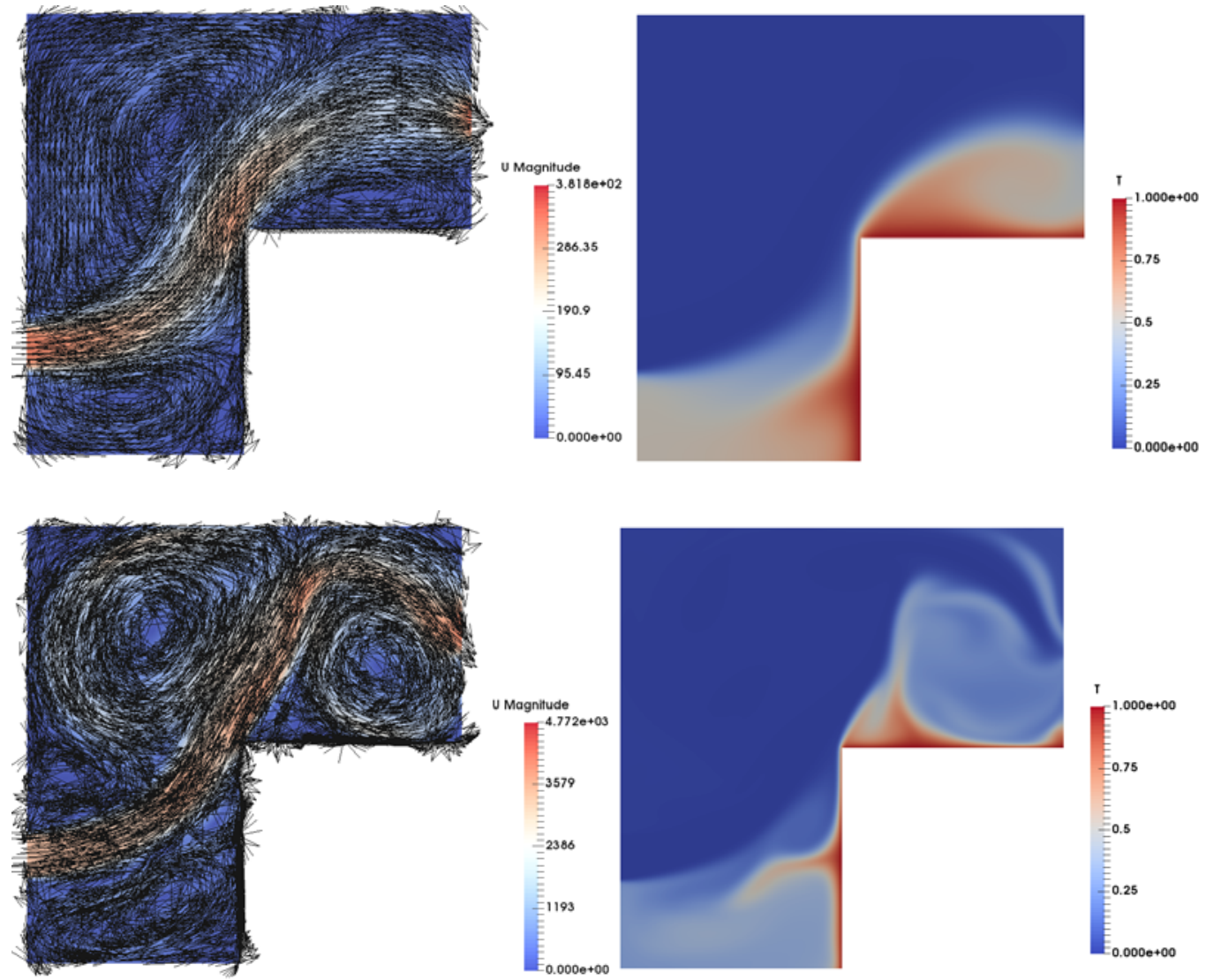

(c)

Figure 14: Snapshots of velocity field (left) and temperature distribution (right) for the case of hybrid forced/buoyancy convection $(R i=1)$, misaligned inflow and outflow sections and cavity with adiabatic bottom wall: a) $R a=10^{4}$, b) $R a=10^{5}$, c) $R a=10^{6}$, d) $R a=10^{7}$. 
Since comparison of the emerging patterns for adiabatic and hot floor also provides important clues regarding the nature of the observed convective structures, we still follow the approach based on a description of the former case followed by comparison with the latter (the impact of such a change on the patterning behavior and evolution towards chaos is summarized in Figs. 14 and 16 for the case with adiabatic and isothermal (hot) floor, respectively).

As evident in Fig. 14(a), for $R a=1 \times 10^{4}$ and adiabatic bottom wall a small velocity roll (close to the floor) is formed in the lower portion of the cavity. The cold fluid enters the inlet with a relatively high velocity and continues to travel straight. It then deflected upward due to continuity effects (fluid must obviously turn around the step in order to reach the outflow section). In this process it is reinforced by buoyancy effects (which tend to propel the relatively warm fluid located in proximity to the vertical wall of the step upwards). It can be seen that the streamlines are relatively regular and give rise to a balloon like structure stretched in the vertical direction, which occupies most of the upper portion of the cavity. Thermal boundary layers are established close to the vertical and horizontal walls of the step, whereas the rest of the cavity is at a moderate temperature. The flow is steady.

Interestingly, for $R a=1 \times 10^{5}$ (Fig. 14(b)) the fluid entering the cavity through the inlet does not spread itself completely in the vertical direction. Indeed, the velocity field does no longer display a vertically stretched balloon like structure. Rather, while some of the fluid is forced out through the outflow section, a certain portion of it is reflected inwards and moves close to the adiabatic ceiling towards the left sidewall giving rise to an extended vortex. The fluid attains its highest velocity values at the inlet, close to the corner of the step and at the outlet.

For $R a=1 \times 10^{6}$ (Fig. 14(c)) no substantial changes can be seen in the structure of the velocity field with respect to $R a=1 \times 10^{5}$. Rather, what distinguishes this specific behavior from the other ones discussed earlier is the increase in the magnitude of flow velocity and strength and vertical size of the roll located just above the step. This increase is indeed responsible for the emergence of the characteristic curl-like shape taken by the thermal plume, which originates from the corner of the step and extends to the right. The flow is steady.

The roll located above the step is further strengthened when the case with $R a=1 \times 10^{7}$ is considered (Fig. 14(d)).

More importantly, as evident in Fig. 14(d), in this case the cold fluid entering the cavity from the left follows a slightly different path. While in the earlier situations, it was flowing along a parabolic route bringing it directly from the inflow section to the outflow one, it now rises continuously until it hits the ceiling (this leading to the formation of a vertically extended thermal plume originating from the corner of the step as evident in the figure). Only after it has collided with the ceiling, this jet can then move again downward and be absorbed by the outflow section. Like the cases treated in Sect. 3.2, the flow is relatively turbulent (see Fig. 15); some localized small eddies develop frequently along the vertical heated wall of the step as witnessed by the presence of small plumes originating from this wall in a direction perpendicular to it (towards the left). Nevertheless, direct comparison with the equivalent case with aligned inflow and outflow sections reveals that in the 
present case the inertial range is less extended (shifted to the right of $10^{4}$, we will come back to this observation later).

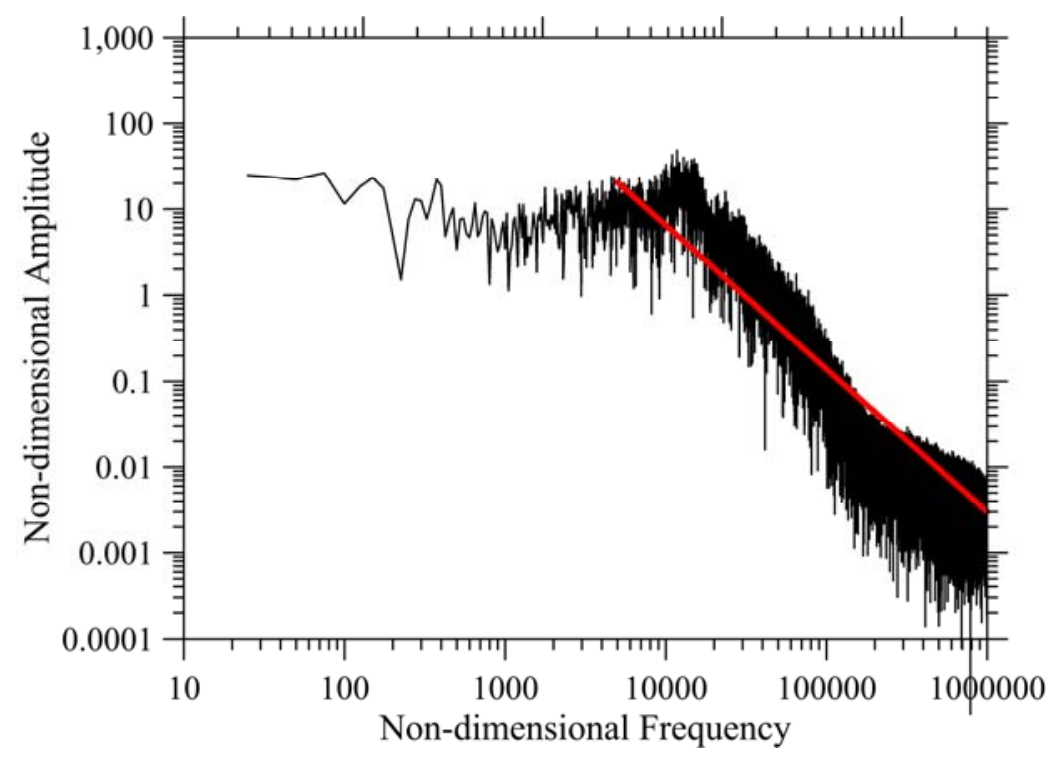

Figure 15: Hybrid convection in cavity with adiabatic bottom wall, $R a=1 \times 10^{7}$ : (a) frequency spectrum related to the velocity signal measured by a numerical probe located at $x=0.25$ and $y=0.75$ (the red line indicates the Kolmogorov scaling law).

Comparison of Table VII with Table V also reveals that the relative importance of $N u_{\text {step }}^{\text {horiz }}$ and $N u_{\text {step }}^{\text {vert }}$ is reverted for the present case ( $N u_{\text {step }}^{\text {horiz }}$ is larger than $N u_{\text {step }}^{\text {vert }}$ when the inflow and outflow sections are not horizontally aligned, whereas the vice versa holds for coaxial sections). An analysis of the flow topology is still useful in this regard. Indeed, we ascribe the notable increase of $N u_{\text {step }}^{\text {horiz }}$ to the specific path taken by the streamlines originating from the inflow section, which always force (regardless of the value of $R a$ ) relatively colder fluid to lick the corner and the hot side of the step.

In the remainder of this section, we finally discuss the situation with the hot bottom (Fig. 16).

For $R a=1 \times 10^{4}$ (Fig. 16(a)) the flow pattern is more or less the same as for adiabatic floor case. A very thick hot boundary layer can be seen covering the floor. For $R a=1 \times 10^{5}$ (Fig. 16(b)) the flow pattern is roughly the same as for the adiabatic floor case (Fig. 14(b)). The flow is still steady. 
Accepted for publication in Int. J. Thermal Science, (ISSN: 1290-0729) on 11 May 2020
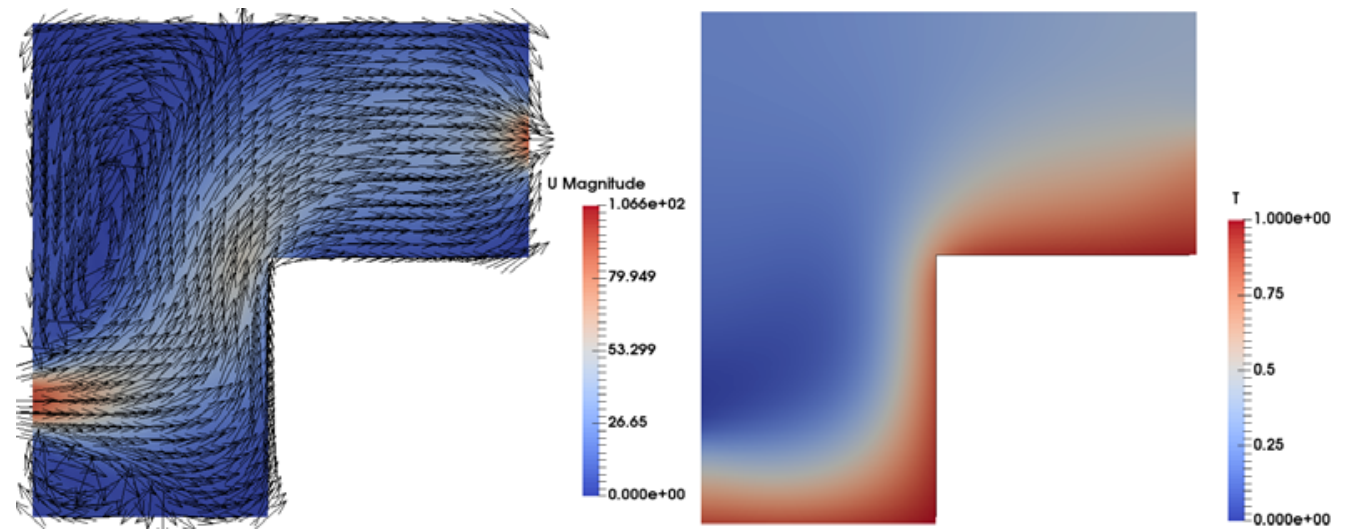

(a)
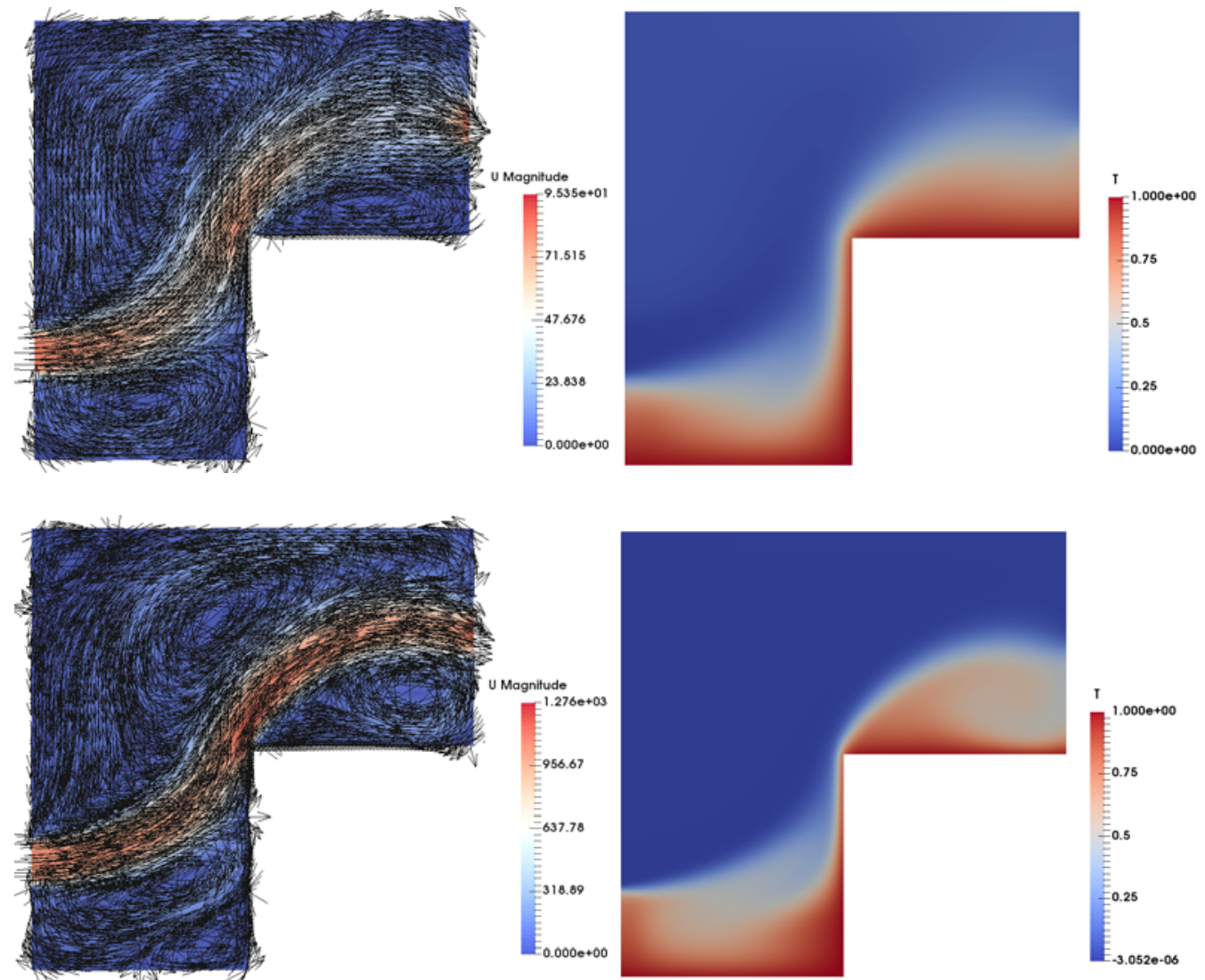

(b)
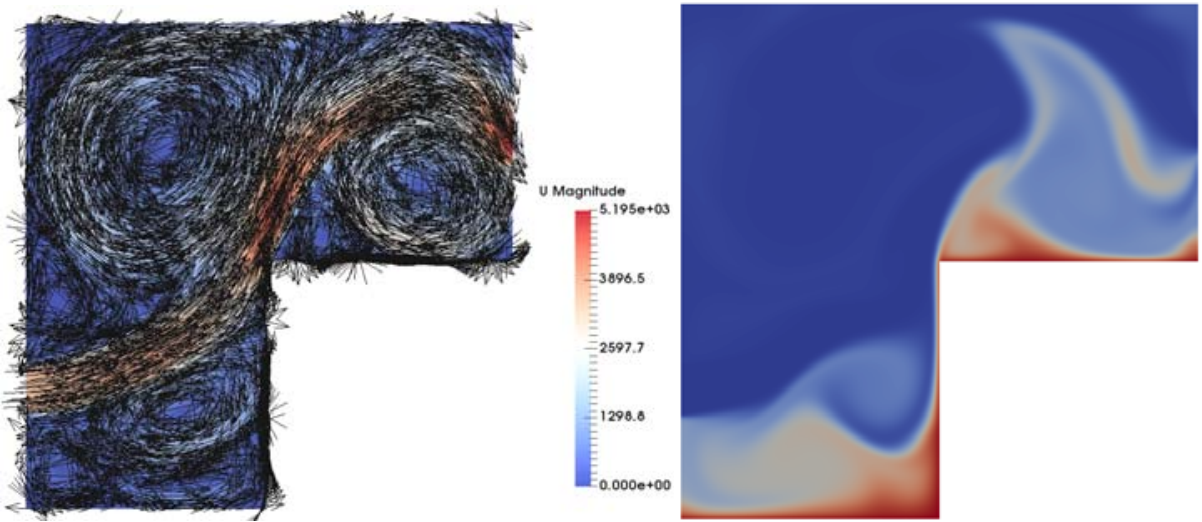

(c)

(d)

Figure 16: Snapshots of velocity field (left) and temperature distribution (right) for the case of hybrid forced/buoyancy convection $(R i=1)$, misaligned inflow and outflow sections and cavity with hot bottom wall: a) $R a=10^{4}$, b) $R a=10^{5}$, c) $R a=10^{6}$, d) $R a=10^{7}$. 
Table VIII: Cases examined for hybrid forced/buoyancy convection $(R i=1)$, misaligned inflow and outflow sections and cavity with hot bottom wall.

\begin{tabular}{cclcc}
\hline \hline$R a$ & Mesh Size & \multicolumn{1}{c}{$N u_{\text {step }}^{\text {horiz }}$} & \multicolumn{1}{c}{$N u_{\text {step }}^{\text {vert }}$} & Regime \\
\hline \hline $1 \times 10^{4}$ & $40 \times 40$ & 4.79508 & 3.2581 & Steady \\
$1 \times 10^{5}$ & $80 \times 80$ & 10.93178 & 4.53742 & Steady \\
$1 \times 10^{6}$ & $120 \times 120$ & 19.7574 & 9.15618 & Steady \\
$1 \times 10^{7}$ & $270 \times 270$ & $\cong 31.9$ (average) & $\cong 33.2$ (average) & Weakly \\
& & & & Turbulent \\
\hline \hline
\end{tabular}

For $R a=1 \times 10^{6}$ (Fig. 16(c)) three velocity rolls can be distinguished. The regular movement of the cold fluid entering the inlet and following a parabolic trajectory until it meets the outflow section is not disturbed in this case (like in the adiabatic floor case). A swirl can yet be seen in the temperature distribution just above the step (compare Figs. 16c and 14c). The flow is steady.

For $R a=1 \times 10^{7}$ (Fig. 16(d)) the behavior is similar to that found for the adiabatic floor case and the same value of $R a$. Like the corresponding circumstances with adiabatic floor, the flow is weakly turbulent (Fig. 17) and characterized by the presence of small eddies located along the horizontal and vertical walls of the step. However, in this case small eddies are also formed along the bottom heated floor (compare Figs. 16d and 14d).

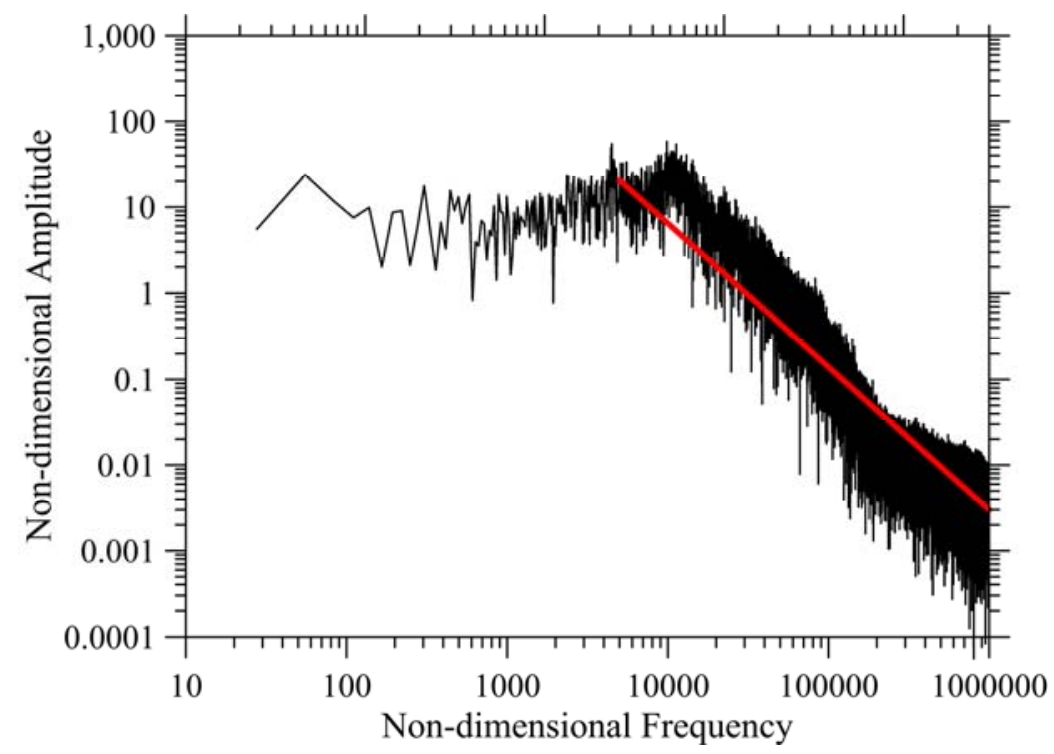

Figure 17: Hybrid convection in cavity with hot bottom wall, $R a=1 \times 10^{7}$ : frequency spectrum related to the velocity signal measured by a numerical probe located at $\mathrm{x}=0.25$ and $\mathrm{y}=0.75$ (the red line indicates the Kolmogorov scaling law). 
Since a steady behavior has been found for $R a<10^{6}$ for both type of thermal conditions assumed for the floor, further analysis was carried out to determine the value of $R a_{\text {critical }}$ for which the flow becomes time-periodic. According to our simulations, such values are $R a_{\text {critical }} \cong 3.8 \times 10^{6}$ and $R a_{\text {critical }} \cong 3.2 \times 10^{6}$, for the cavity with adiabatic and isothermal (hot) floor, respectively, which indicate that in these cases an increase in buoyancy has a slightly destabilizing influence.

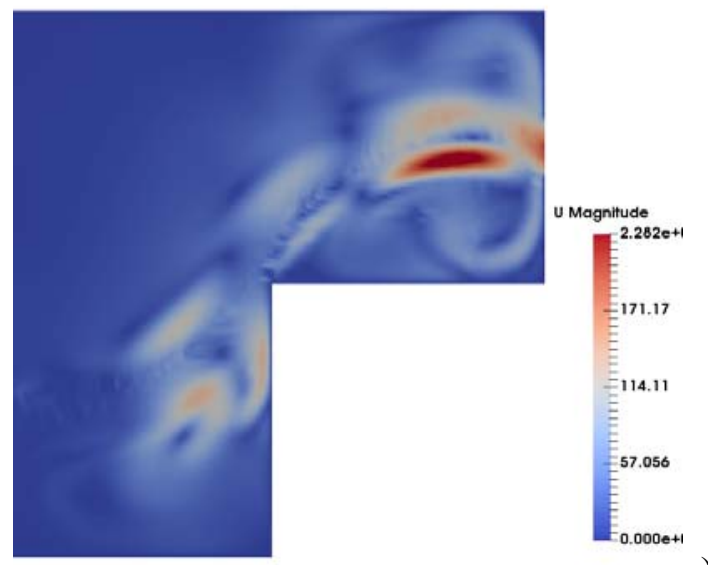

a)

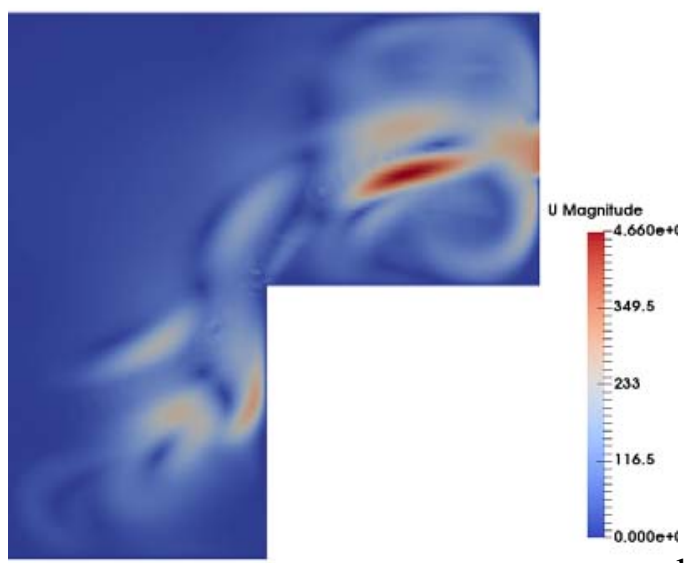

b)

Figure 18: Snapshot of velocity disturbances for the case of hybrid forced/buoyancy convection $(R i=1)$, misaligned inflow and outflow sections: a) cavity with adiabatic floor, $\left.R a=3.8 \times 10^{6} ; \mathrm{b}\right)$ cavity with hot floor, $R a=3.2 \times 10^{6}$.

The small difference in the critical value of the Rayleigh number is in line with the behavior of the disturbances, which as shown in Fig. 18 display a relatively similar configuration for both cases. In particular, they exhibit a similar magnitude and are concentrated along the main path taken by the cold fluid (entering the cavity from the left) to reach the outflow section. Among other things, this figure is also useful as it reveals that the instability is still of a shear-driven nature. Apart from the region located above the step, disturbances are also significant in the left lower portion of the cavity where their presence can directly be ascribed to the strong horizontal shear stress that is attained there as a result of the curvature of the main current. This can be seen as an important distinguishing mark with respect to the perturbation patterns seen in Figs. 13 where disturbances were more concentrated in the portion of the domain located just above the step (where vertical shear stresses are high).

Comparison with the configuration with horizontally aligned inflow and outflow sections also leads to the conclusion that a decrease in the height (distance from the floor) of the inflow section can have a stabilizing influence on the flow (the $R a_{\text {critical }}$ for misaligned sections being approximately three times that needed to induce time-dependent flow in the situation with coaxial sections, which also explains why in the present case the inertial range is shifted to the right in both Figs. 15 and 17). Further insights into the thermal behavior can finally be gained by comparing the values of $N u_{\text {step }}^{\text {horiz }}$ in Tables VII and VIII. We ascribe the non-negligible shrinkage in the values of $N u_{\text {step }}^{\text {horiz }}$ for the case with hot floor and moderate values of $R a$, to the effect that such boundary exerts on the cold fluid 
entering from the left side (causing a quick rise in the temperature of such fluid thereby mitigating its ability to extract heat from the top surface of the step).

\section{Conclusions}

When pure buoyancy is considered in a cavity with a square hot obstruction on the bottom, multicellular configurations are produced with number of rolls changing according to the value of the Rayleigh number. For relatively high values of $R a$, these multicellular systems are driven by the development of spatially extended thermal plumes in the system, which tend to originate from the corner of the obstruction and assume different inclination according to the considered condition (switching from an almost horizontal or slightly inclined configuration for the case with adiabatic bottom to an almost vertical state when the bottom is kept at a fixed hot temperature). Most interestingly, for these cases, heat islands can be produced in some circumstances. For pure buoyancy these are always located in the lower part of the domain, which can be ascribed to the insufficient mixing between the upper and lower fluid regions that is established when the flow pattern takes a specific topological configuration (at relatively high values of $R a$ ).

In a rather unexpected way, replacement of the adiabatic bottom with an isothermal wall, leads to a stabilization of the flow field due the non-trivial coupling established in the pure buoyancy case between the flow of Hadley type (which develops from the vertical side of the obstruction) and that of RB nature, driven by horizontal hot boundaries.

When hybrid convection (with unit value of the Richardson number) is considered, a variety of other patterns emerge, corresponding to different possible realizations of the route that cold fluid can take to reach the outlet. These possible multiple paths follow naturally from the relative position of the inlet with respect to the floor and the rich variety of possible interactions that can be established between the cold fluid injected in the cavity and the buoyancy flow of thermal nature originating from the hot surfaces. The latter can support or oppose to the transport of fluid from the inflow to the outflow section depending on the considered circumstances.

Changing the position of the inflow section can also have remarkable effects on such dynamics. Heat islands that, for the case of inflow section horizontally aligned with the outflow section, are formed in the lower portion of the domain (as in the pure buoyancy case), disappear when the inflow section is close to the bottom wall. A change in the position of the inflow section can also affect in an appreciable way the route towards time-dependence and chaos and how this, in turn, is influenced by the nature of the bottom wall (being it adiabatic or isothermal). While, in general, for hybrid convection, a switch from the adiabatic to the isothermal boundary condition causes a stabilization of the flow, the effective change experienced by the critical Rayleigh number strongly depends on the location of the inflow section.

Future studies shall be devoted to extend the present analysis assuming different aspect ratio of the cavity, different relative thickness of the step, different vertical extension of the inflow and outflow 
sections (corresponding to other volumetric flow rates of the cold fluid), and eventually threedimensional configurations.

\section{References}

[01] Lappa M., (2009), Thermal Convection: Patterns, Evolution and Stability, John Wiley \& Sons, Ltd (2009, Chichester, England).

[02] Hattori H. and Nagano Y., (2010), Investigation of turbulent boundary layer over forward facing step via direct numerical simulation, Int. J. Heat Fluid Flow, 31, 284-294.

[03] Dai Z., Li T., Zhang W., Zhang J., (2020), Numerical Study on Aerodynamic Performance of High-Speed Pantograph with Double Strips, Fluid Dynamics \& Materials Processing, 16 (1), 31-40. [04] Redchyts D.O., Shkvar E.A., Moiseienko S.V., (2020), Computational Simulation of Turbulent Flow Around Tractor-Trailers, Fluid Dynamics \& Materials Processing, 16(1), 91-103.

[05] Sherry M., Jacono D.L., Sheridan J., (2010), An experimental investigation of the recirculation zone formed downstream of a forward facing step, J. Wind Eng. Ind. Aerod., 98, 888-894.

[06] Hillier R. and Cherry N., (1981), The effects of stream turbulence on separation bubbles, Journal of Wind Engineering and Industrial Aerodynamics 8, 49-58.

[07] Houde H., Lu J. and Bao W., (1994), A Discrete Artificial Boundary Condition for Steady Incompressible Viscous Flows in a No-Slip Channel Using a Fast Iterative Method, J. Comput. Phys., 114, 201-208.

[08] Stüer H., Gyr A., Kinzelbach W., (1999), Laminar separation on a forward facing step, Eur. J. Mech. B/Fluids 18, 675-692.

[09] Barbosa-Saldaña J.G. and Anand N. K., (2007), Flow Over a Three-Dimensional Horizontal Forward-Facing Step, Numerical Heat Transfer, Part A: Applications, 53(1),1-17.

[10] Abdalla I. E., Yang Z. and Cook M., (2009), Computational analysis and flow structure of a transitional separated-reattached flow over a surface mounted obstacle and a forward-facing step, International Journal of Computational Fluid Dynamics, 23(1), 25-57, DOI: 10.1080/10618560802566246

[11] Zhu H. and Fu S., (2017), Forward-facing steps induced transition in a subsonic boundary layer, Science China, Physics, Mechanics \& Astronomy, 60(10): 104712 - doi: 10.1007/s11433-0179069-3.

[12] Graziani A., Lipperta M., Uystepruyst D., Keirsbulck L., (2017), Scaling and flow dependencies over forward-facing steps, International Journal of Heat and Fluid Flow 67, 220-229 [13] Kiya M. and Sasaki K., (1983), Structure of a turbulent separation bubble, J. Fluid Mech., 137, $83-113$.

[14] Cherry N.J., Hillier R., Latour M.E., (1985), Unsteady measurements in a separated and reattaching flow, J. Fluid Mech. 144, 13-46.

[15] Largeau J.F. and Moriniere V., (2007), Wall pressure fluctuations and topology in separated flows over a forward-facing step, Exp Fluids, 42, 21-40.

[16] Camussi R., Felli M., Pereira F., Aloisio G., Di Marco A., (2008), Statistical properties of wall pressure fluctuations over a forward-facing step, Phys. Fluids, 20, 075113 (13 pp).

[17] Sun Z. and Jaluria Y., (2011), Conjugate thermal transport in gas flow in long rectangular microchannel, J. Electron. Packag. 133(2), 021008 (11 pages).

[18] Arrif T.,, Chehhat A., Abo-Serie E., Benchabane A., (2018), Numerical Study of Natural Convection in Square Tilted Solar Cavity Considering Extended Domain, Fluid Dynamics \& Materials Processing, 14(4), 223-242.

[19] Nadjib H., Adel S., Djamel S., Abderrahmane D., (2018), Numerical Investigation of Combined Surface Radiation and Free Convection in a Square Enclosure with an Inside Finned Heater, Fluid Dynamics \& Materials Processing, 14(3), 155-175. 
[20] Lappa M., (2019), On the Highly Unsteady Dynamics of Multiple Thermal Buoyant Jets in Cross Flows, Phys. Fluids, 31, 115105 (27 pages).

[21] Abu-Mulaweh H.I., Armaly B.F., Chen T.S., (1993), Measurements of laminar mixed convection flow over a horizontal forward-facing step, J. Thermophys. Heat Transfer, 7, 569-573.

[22] Abu-Mulaweh H.I., (2003), A review of research on laminar mixed convection flow over backward- and forward-facing steps, Int. J. Therm. Sci., 42(9), 897-909.

[23] Moosavi R. and Nassab A.G., (2008), Turbulent forced convection over a single inclined forward step in a duct: part I-flow field, Eng Appl Comp Fluid, 2, 366-374.

[24] Nassab S.A.G., Moosavi R., Sarvari S.M.H., (2009), Turbulent forced convection flow adjacent to inclined forward step in a duct, Int. J. Therm. Sci., 48, 1319-1326.

[25] Oztop H.F., Mushatet K. S., Y1lmaz İ., (2012), Analysis of turbulent flow and heat transfer over a double forward facing step with obstacles, International Communications in Heat and Mass Transfer, 39,1395-1403.

[26] Xie W.A. and Xi G.N., (2017), Geometry effect on flow fluctuation and heat transfer in unsteady forced convection over backward and forward facing steps, Energy, 132, 49-56.

[27] Lappa M., (2017), On the oscillatory hydrodynamic modes in liquid metal layers with an obstruction located on the bottom, Int. J. Therm. Sci., 118, 303-319. DOI: 10.1016/j.ijthermalsci.2017.04.013

[28] Lappa M., (2019a), On the gravitational suppression of hydrothermal modes in liquid layers with a blockage on the bottom wall, Int. J. Therm. Sci., , 145, November 2019, 105987 (19 pages)

[29] Khatra L., El Qarnia H., El Ganaoui M., (2019), The Effect of the Fin Length on the Solidification Process in a Rectangular Enclosure with Internal Fins, Fluid Dynamics \& Materials Processing, 15(2), 125-137.

[30] Yarin L.P., (2009) Cooling Systems of Electronic Devices. In: Fluid Flow, Heat Transfer and Boiling in Micro-Channels. Heat and Mass Transfer. Springer, Berlin, Heidelberg

[31] Venkatasubbaiah K. and Jaluria Y., (2012), Numerical simulation of enclosure fires with horizontal vents, Numer. Heat Tran. 62, 179-196.

[32] Morsli S., Boussoufi M., Sabeur A., El Ganaoui M., Bennacer R., (2018) Small to large scale mixed turbulent convection: buildings application, Int. J. Numer. Methods Heat Fluid Flow, 28(1), 188-205, https://doi.org/10.1108/HFF-10-2017-0392

[33] Kachi S., Bensouici F.Z., Ferroudj N. and Boudebous S., (2019), Effect of Richardson Number on Unsteady Mixed Convection in a Square Cavity Partially Heated From Below, Fluid Dynamics \& Materials Processing, 15(2), 89-105. doi:10.32604/fdmp.2019.00263

[34] Gollub J.P. and Benson S.V., (1980), Many Routes to Turbulent Convection, J. Fluid Mech., $100,449-470$.

[35] Gershuni G.Z., Laure P., Myznikov V.M., Roux B., Zhukhovitsky E.M., (1992), On the stability of plane-parallel advective flows in long horizontal layers, Microgravity Q., 2(3), 141-151.

[36] Hattori T , Norris S , Kirkpatrick M , Armfield S., (2013), Comparison of non-reflective boundary conditions for a free-rising turbulent axisymmetric plume, Int. J. Numer. Methods Fluids, 72(12), 1307-1320.

[37] Dong S., Karniadakis G.E., Chryssostomidis C., (2014), A robust and accurate outflow boundary condition for incompressible flow simulations on severely-truncated unbounded domains, J. Comput. Phys., 261, 83-105.

[38] Issa R.I.,(1986), "Solution of the implicitly discretized fluid flow equations by operatorsplitting", J. Comp. Physics, 62, 40-65.

[39] Ladyzhenskaya O.A., (1969), The Mathematical Theory of Viscous Incompressible Flow, Gordon and Breach, 2nd Edition, New York - London, 1969.

[40] Gresho P.M., Sani R.T., (1987), On pressure boundary conditions for the incompressible Navier-Stokes equations, Int. J. Numer. Meth. Fluids, 7, 1111-1145.

[41] Guermond J.-L. and Quartapelle L., (1998), On stability and convergence of projection methods based on pressure Poisson equation, Int. J. Numer. Meth. Fluids, 26, 1039-1053. 
[42] Guermond J.-L., Minev P. and Shen J., (2006), An Overview of Projection Methods for Incompressible Flows. Comput. Methods, Comput. Methods Appl. Mech. Eng., 195, 6011-6045.

[43] Lappa M., (1997), "Strategies for parallelizing the three-dimensional Navier-Stokes equations on the Cray T3E"; Science and Supercomputing at CINECA, 11, 326-340. ISBN-10: 88-86037-031, M. Voli, Editor (Bologna, Italy).

[44] Lappa M., (2016), A Mathematical and Numerical Framework for the Analysis of Compressible Thermal Convection in Gases at very high Temperatures, Journal of Computational Physics, 313, 687-712.

[45] Rhie C.M. and Chow W.L., (1983), Numerical study of the turbulent flow past an airfoil with trailing edge separation, AIAA J 21,1525-1532

[46] Ouertatani N., Cheikh N. B., Beya B.B., Lili T., (2008), "Numerical simulation of twodimensional Rayleigh-Benard convection in an enclosure", C.R. Mecanique, 336(5), 464-470.

[47] De Vahl Davis and Jones I. P., (1983), "Natural convection in a square cavity a comparison exercise", International journal for numerical methods in fluids, vol. 3, pp. 227-248.

[48] Pope S.B. (2000), Turbulent Flows, Cambridge University Press, 2000

[49] Coceal O., Thomas T.G., Castro I.P. , Belcher S. E., (2006), Mean flow and turbulence statistics over groups of urban-like cubical obstacles, Boundary-Layer Meteorol., 121, 491-519

[50] Kerr R.M., (1996), Rayleigh number scaling in numerical convection, J. Fluid Mech. 310, 139179.

[51] De A.K., Eswaran V., Mishra P.K., (2017), Scalings of heat transport and energy spectra of turbulent Rayleigh-Bénard convection in a large-aspect-ratio box, Int. J. Heat Fluid Flow, 67, 111124.

[52] Paolucci S., (1990), Direct numerical simulation of two-dimensional turbulent natural convection in an enclosed cavity, J. Fluid Mech., 215, 229-262.

[53] Farhangnia M., Biringen S, Peltier L. J., (1996), Numerical Simulation of Two-dimensional Buoyancy-driven Turbulence in a Tall Rectangular Cavity, Int. J. Numer. Meth. Fluids, 23(12), $1311-1326$.

[54] Lappa M. and Gradinscak T., (2018), On the Oscillatory Modes of Compressible Thermal Convection in inclined differentially heated cavities, Int. J. of Heat and Mass Transfer, 121, 412436.

[55] Hier Majumder C. A., Yuen D. A. and Vincent A., (2004), Four dynamical regimes for a starting plume model, Phys. Fluids, 16(5), 1516-1531.

[56] Napolitano L.G., (1982), Surface and Buoyancy Driven Free Convection, Acta Astronautica, 9(4), 199-215.

[57] Lappa M., (2011), Some considerations about the symmetry and evolution of chaotic Rayleigh-Bénard convection: The flywheel mechanism and the "wind" of turbulence, Comptes Rendus Mécanique, 339, 563-572. DOI:10.1016/j.crme.2011.05.002

[58] Oztop H.F., Dagtekin I., Bahlou A., (2004), Comparison of position of a heated thin plate located in a cavity for natural convection, Communications in Heat and Mass Transfer, 31(1), 121132.

[59] Saravanana S., Abdul Hakeem A.K., Kandaswamy P., Leeb J., (2008), Buoyancy convection in a cavity with mutually orthogonal heated plates. Computers and Mathematics with Applications, 55(12), 2903-2912.

[60] Saravanan S. and Sivaraj C. (2013), Combined natural convection and thermal radiation in a square cavity with a nonuniformly heated plate, International Journal of Heat and Fluid Flow, 40, 54-64.

[61] Nadjib H., Adel S., Djamel S. and Abderrahmane D., (2018), Numerical Investigation of Combined Surface Radiation and Free Convection in a Square Enclosure with an Inside Finned Heater, Fluid Dynamics \& Materials Processing, 14(3), 155-175. 
[62] Yu H., Li N., and Ecke R.E., (2007), Scaling in laminar natural convection in laterally heated cavities: Is turbulence essential in the classical scaling of heat transfer?, Phys Rev E, 76, 026303 (4 pages).

[63] Corvaro F., and Paroncini M., (2007), Experimental analysis of natural convection in square cavities heated from below with 2D-PIV and holographic interferometry techniques, Experimental Thermal and Fluid Science 31, 721-739.

[64] Stevens R.J. A. M., Verzicco R., and Lohse D., (2010), Radial boundary layer structure and Nusselt number in Rayleigh-Bénard convection, J. Fluid Mech., 643, 495-507.

[65] Goldhirsch I., Pelz R. B. and Orszag S. A., (1989), "Numerical simulation of thermal convection in a two-dimensional finite box", J. Fluid Mech., 199, 1-28.

[66] Bouabdallah S., Ghernaout B., Teggar M., Benchatti A. and Benarab F-Z., (2016), Onset of Natural Convection and Transition Laminar-Oscillatory Convection Flow in Rayleigh-Bénard Configuration, Int. J. Heat Technol., 34(1), 151-157. DOI: 10.18280/ijht.340122

[67] Paolucci S. and Chenoweth D.R., (1989), "Transition to chaos in a differentially heated vertical cavity", J. Fluid Mech., 201, 379-410.

[68] Le Quéré P. and Behnia M., (1998), "From onset of unsteadiness to chaos in a differentially heated square cavity", J. Fluid Mech., 359, 81-107.

[69] Kolmogorov, A.N. (1941a) The local structure of turbulence in incompressible viscous fluids at very large Reynolds numbers, Dokl. Akad. Nauk. SSSR 30, 299-303. Reprinted in Proc. R. Soc. London A 434, 9-13 (1991).

[70] Kolmogorov, A.N. (1941b) On the degeneration of isotropic turbulence in an incompressible viscous fluids, Dokl. Akad. Nauk. SSSR 31, 538-541.

[71] Kolmogorov, A.N. (1941c) Dissipation of energy in isotropic turbulence. Dokl. Akad. Nauk. SSSR, 32, 19-21.

[72] Kolmogorov, A.N. (1942) Equations of turbulent motion in an incompressible fluid. Izv. Akad. Nauk. SSSR ser. Fiz. 6, 56-58.

[73] Kraichnan R.H., (1974), On Kolmogorov's inertial-range theories, J. Fluid Mech., 62, 305-330

[74] Pera L. and Gebhart B., (1971), On the stability of laminar plumes: Some numerical solutions and experiments, Int. J. Heat Mass Transfer, 14(7), 975-982.

[75] Desrayaud G. and Lauriat G., (1993), Unsteady Confined Buoyant Plumes, J. Fluid Mech., $252,617-646$.

[76] Cortese T. and Balachandar S., (1993), Vortical nature of thermal plumes in turbulent convection, Phys. Fluids A, 5, 3226-3232.

[77] Vincent A.P. and Yuen D.A., (1999), Plumes and waves in two-dimensional turbulent thermal convection, Phys. Rev. E 60(3), 2957-2963.

[78] Ali S.Z and Dey S., (2017), Hydrodynamic instability of meandering channels, Phys. Fluids, $29,125107$.

[79] Hemchandra S., Shanbhogue S., Hong S., and Ghoniem A.F., (2018), Role of hydrodynamic shear layer stability in driving combustion instability in a premixed propane-air backward-facing step combustor, Phys. Rev. Fluids, 3, 063201 (20 pp). 